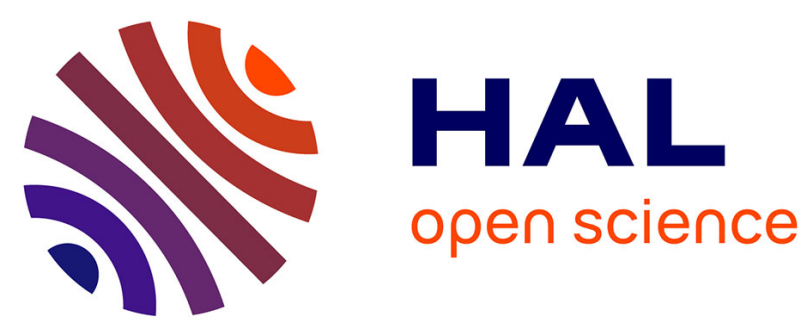

\title{
The Seismic anisotropy of the Earth's mantle: From single crystal to polycrystal
}

D Mainprice, Guilhem Barruol, Walid Ben Ismaï

\section{To cite this version:}

D Mainprice, Guilhem Barruol, Walid Ben Ismaill. The Seismic anisotropy of the Earth's mantle: From single crystal to polycrystal. Shun-Ichiro Karato; Alessandro Forte; Robert Liebermann; Guy Masters; Lars Stixrude. Earth's Deep Interior: Mineral Physics and Tomography From the Atomic Scale to the Global Scale, 117, American Geophysical Union, pp.237 - 264, 2000, Geophysical Monograph Series, 9780875909752. 10.1029/GM117p0237 . hal-01391524

\section{HAL Id: hal-01391524 \\ https://hal.univ-reunion.fr/hal-01391524}

Submitted on 14 Nov 2016

HAL is a multi-disciplinary open access archive for the deposit and dissemination of scientific research documents, whether they are published or not. The documents may come from teaching and research institutions in France or abroad, or from public or private research centers.
L'archive ouverte pluridisciplinaire $\mathbf{H A L}$, est destinée au dépôt et à la diffusion de documents scientifiques de niveau recherche, publiés ou non, émanant des établissements d'enseignement et de recherche français ou étrangers, des laboratoires publics ou privés. 


\section{HAL \\ HAL}

\section{The Seismic anisotropy of the Earth's mantle: From single crystal to polycrystal \\ D Mainprice, Guilhem Barruol, Walid Ben Ismaïl}

\section{To cite this version:}

D Mainprice, Guilhem Barruol, Walid Ben Ismaill. The Seismic anisotropy of the Earth's mantle: From single crystal to polycrystal. Shun-Ichiro Karato, Alessandro Forte, Robert Liebermann, Guy Masters, Lars Stixrude. Earth's Deep Interior: Mineral Physics and Tomography From the Atomic Scale to the Global Scale, 117, American Geophysical Union, pp.237 - 264, 2000, Geophysical Monograph Series, 9780875909752. <10.1029/GM117p0237>. <hal$01391524>$

\section{HAL Id: hal-01391524 \\ http://hal.univ-reunion.fr/hal-01391524}

Submitted on 3 Nov 2016

HAL is a multi-disciplinary open access archive for the deposit and dissemination of scientific research documents, whether they are published or not. The documents may come from teaching and research institutions in France or abroad, or from public or private research centers.
L'archive ouverte pluridisciplinaire HAL, est destinée au dépôt et à la diffusion de documents scientifiques de niveau recherche, publiés ou non, émanant des établissements d'enseignement et de recherche français ou étrangers, des laboratoires publics ou privés. 


\title{
The Seismic Anisotropy of the Earth's Mantle: From Single Crystal to Polycrystal
}

\author{
D. Mainprice, G. Barruol and W. Ben Ismaïl
}

Laboratoire de Tectonophysique, CNRS, Université de Montpellier II, Montpellier, France.

The anisotropic single crystal seismic properties are reviewed in the light of recent experimental and theoretical determinations. Although considerable progress has been made on the determination of single crystal properties, data are still lacking, particularly for the temperature derivatives of transition zone and lower mantle phases. The common types of LPO of olivine, opx and cpx are presented together with their associated seismic properties. It is emphasized that simple seismic symmetry pattern of upper mantle rocks are a direct result of the interaction of olivine, opx and cpx. Using the standard structural frame ( $\mathrm{X}$ lineation, $\mathrm{Z}$ pole to foliation) for typical LPOs of olivine, opx and cpx have the maximum Vp parallel to $\mathrm{X}$, in the $\mathrm{XZ}$ plane and parallel to $\mathrm{Y}$ respectively. Destructive interference occurs between these minerals and hence P-wave anisotropy should be sensitive to the aggregate composition. For shear wave splitting (dVs) typical olivine and opx LPOs result in similar patterns with the maximum $\mathrm{dVs}$ in the $\mathrm{YZ}$ plane and the fast split shear wave (Vs1) polarized parallel to the foliation. A typical cpx LPO on the other hand produces destructive interference as the max $d V s$ is close to $X$. By comparison with experiments and numerical simulations, it is estimated that upper mantle samples have an olivine LPO strength which recorded shear strain gamma of between 0.25 and 2.0. Pyrolite and piclogite models are compared with global transverse isotropic models. The slowly reducing P-wave anisotropy in the first $200 \mathrm{~km}$ can be explained by a model with constant composition and LPO strength. The sharp decrease in the observed anisotropy in the global models cannot be explained by the transformation of opx to cpx at $300 \mathrm{~km}$, it is proposed that this decrease is due to a reduction in LPO strength from 200 to $350 \mathrm{~km}$ at the base of the lithosphere.

\section{INTRODUCTION}

Seismic anisotropy is a characteristic feature of the Earth, with anisotropy being present near the surface due to aligned cracks (e.g. Crampin, 1984), in the lower crust, upper and lower mantle due to mineral preferred orientation (e.g. Mainprice and Nicolas, 1989, Karato, 1998). At the bottom of the lower mantle (D" layer, e.g. Kendall and Silver, 1998) and in the solid core (e.g. Morelli et al., 1986) 


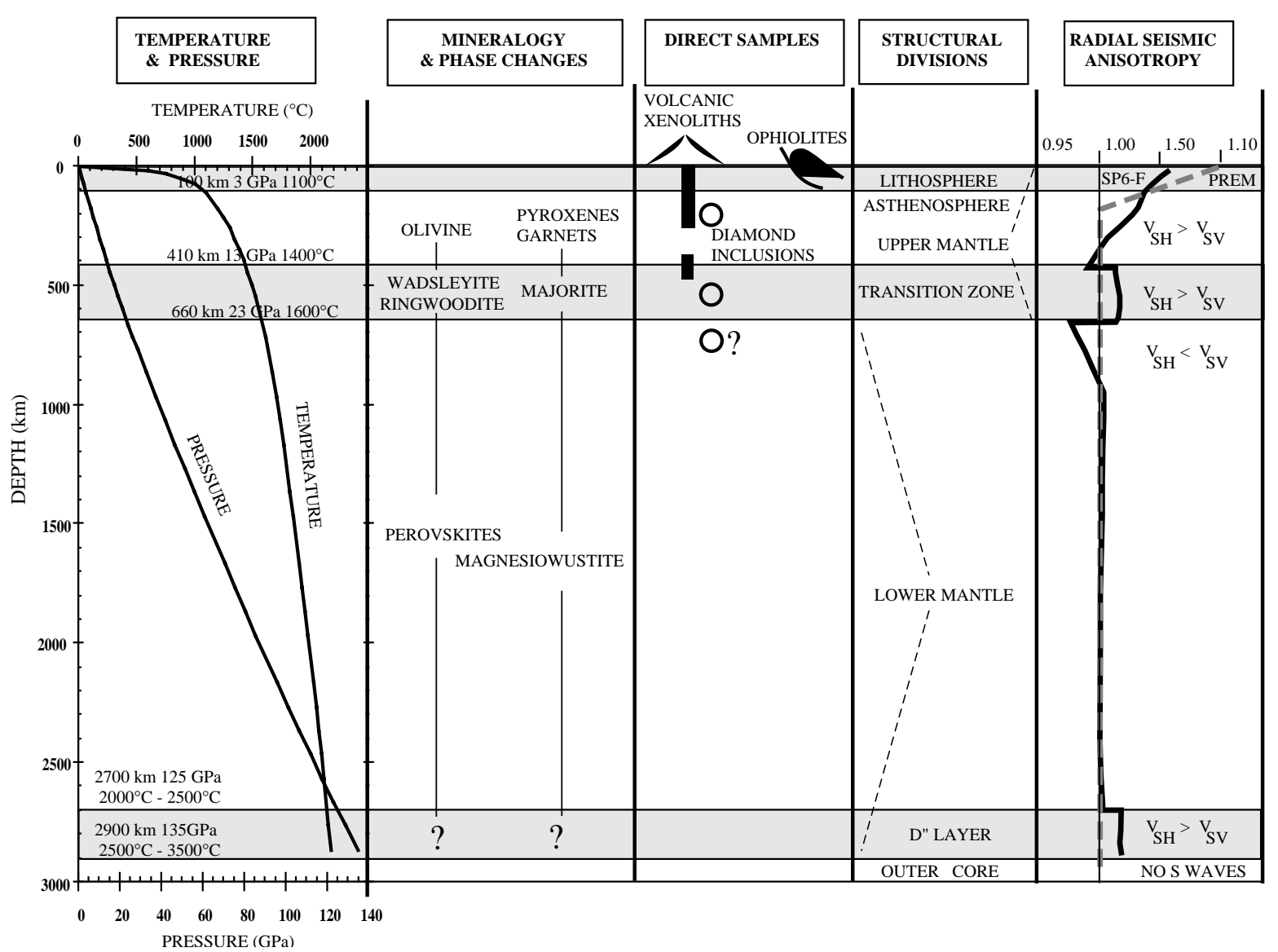

Figure 1. The major physical and chemical variations in the Earth's mantle which control seismic anisotropy partly inspired after Gillet (1995). The seismic model SP6-F comes from Montagner and Kennett (1996).

the causes of anisotropy are still controversial (Fig. 1). In some cases, multiple physical factors could be contributing to the measured anisotropy, for example mineral preferred orientation and alignment of melt inclusions at mid-ocean ridge systems (Mainprice, 1997). In the upper mantle, the pioneering work of Hess (1964) and Raitt et al. (1969) from Pn velocity measurements in the shallow mantle of the ocean basins showed azimuthal anisotropy. Long-period surface waves studies (e.g. Nataf et al., 1984, Montagner and Tanimoto 1990) have since confirmed that azimuthal and $\mathrm{SH} / \mathrm{SV}$ polarization anisotropy are global phenomena in the Earth's upper mantle, particularly in the top $200 \mathrm{~km}$ of the sub-continental mantle. Anisotropic global tomography, based mainly on surface wave data, has shown that anisotropy is very strong in the sub-continental mantle and present generally in the upper mantle but significantly weaker at greater depths. The large wavelengths used in longperiod surface waves studies means that such methods are insensitive to heterogeneity less than the wavelength of about $1500 \mathrm{~km}$. More recently in an effort to address the problem of regional variations of anisotropy the splitting of SKS teleseismic shear waves which propagate vertically have been extensively used (Kind et al., 1985, Silver and Chan 1988, 1991; Vinnik et al., 1989). At continental stations, SKS studies show that the azimuth of the fast polarization direction is frequently parallel to the trend of mountain belts (see reviews from Silver, 1996 and Savage, 1999). From the earliest observations, it was clear that the anisotropy in the upper mantle was caused by the preferred orientation of olivine crystals induced by plastic deformation related to mantle flow processes at the geodynamic or plate tectonic scale.

The major cause of seismic anisotropy in the upper mantle is the crystal preferred orientation caused by plastic deformation. Knowledge of the lattice preferred orientation (L.P.O.) and its evolution requires well characterized naturally deformed samples, experimentally deformed samples and numerical simulation for more complex deformation histories of geodynamic interest. The crystal preferred orientation not only causes seismic anisotropy, but also records some aspects of the deformation history. Samples of the Earth's mantle are readily found on the surface in the form of ultra-mafic massifs, xenoliths in basaltic or kimberlitic volcanics and as inclusions in diamonds (Fig. 1). However, samples from depths greater than $220 \mathrm{~km}$ are extremely rare. Upper mantle samples large enough for the measurement of LPO have been recovered from kimberlitic volcanics in South Africa to a depth of 
about $220 \mathrm{~km}$ established by geobarometry (e.g. Boyd, 1973). Kimberlite mantle xenoliths of deeper origin (>300 $\mathrm{km}$ ) with evidence for equilibrated majorite garnet which is now preserved as pyrope garnet with exsolved pyroxene have been reported (Haggerty and Sautter, 1990; Sautter et al., 1991). Samples of even deeper origin are preserved as inclusions in diamonds. Although most diamonds crystallize at depths of $150-200 \mathrm{~km}$, some diamonds contain inclusions of majorite (Moore and Gurney, 1985), enstatite and magnesiowüstite (Scott-Smith et al., 1984) and $\mathrm{CaSiO}_{3}+(\mathrm{Fe}, \mathrm{Mg}) \mathrm{SiO}_{3}+\mathrm{SiO}_{2}$ (Harte and Harris, 1993). The mineral associations imply transition zone $(410-660 \mathrm{~km})$ and lower mantle origins for these diamond inclusions (Kesson and Fitz Gerald, 1991). Although these samples help to constrain mantle petrology, they are too small to provide information about LPO. Hence knowledge of LPO in the transition zone and lower mantle will be derived from deformation experiments at high pressure and temperature (e.g. Sharp et al., 1994; Dupas-Bruzek et al., 1998; Karato et al., 1998).

It has been accepted since the PREM seismic model (Dziewonski and Anderson, 1981) that the top $200 \mathrm{~km}$ of the Earth's mantle is anisotropic on a global scale (Fig. 1). Indeed, a seismic discontinuity at about $200 \mathrm{~km}$ was first reported by Lehmann $(1959,1961)$ which now bears her name. However, the discontinuity is not always present at the same depth. Anderson (1979) interpreted the discontinuity as the petrological change of garnet lherzolite to eclogite. More recently, interpretations have favored an anisotropy discontinuity, either a local anisotropic decoupling shear zone marking the base of the lithosphere (Leven et al., 1981), a transition from an anisotropic mantle deforming by dislocation creep to isotropic mantle undergoing diffusion creep (Karato, 1992) or simply the base of an anisotropic layer beneath continents (Gaherty and Jordan, 1995). Global tomography studies show that the base of the anisotropic sub-continental mantle may vary in depth from 100 to $450 \mathrm{~km}$ (e.g. Polet and Anderson, 1995).

A major seismic discontinuity at $410 \mathrm{~km}$ is due to the transformation of olivine to wadsleyite (e.g. Helffrich and Wood, 1996) with a shear wave impedance contrast of $6.7 \%$ (e.g. Shearer, 1996). The $410 \mathrm{~km}$ discontinuity has a topography within $5 \mathrm{~km}$ of the global average. The olivine to wadsleyite transformation will result in the lowering of anisotropy with depth (see Table 1). Global tomography models (e.g. Montagner, 1994; Montagner and Kennett, 1996) indicate that the strength of anisotropy is less in the transition zone $(410 \mathrm{~km}$ to $660 \mathrm{~km})$ than in the upper mantle (Fig. 1) which is in accordance with the increase in nearly isotropic phases such as garnet, majorite and ringwoodite in the transition zone (Table 1). A weaker discontinuity with a shear wave impedance contrast of $2.9 \%$ has been reported by Shearer and co-workers (e.g. Shearer 1996; Flanagan and Shearer, 1998) at $520 \mathrm{~km}$. The discontinuity at $520 \mathrm{~km}$ depth has been attributed to the wadsleyite to ringwoodite transformation by Shearer (1996) and to exsolution of Ca-perovskite from majorite garnet (Ita and Stixrude, 1992). The wadsleyite to ringwoodite transformation results in the lowering of anisotropy with depth (see Table 1) in the lower part of the transition zone, whereas the exsolution of Ca-perovskite from majorite garnet would result in an increase. Recent seismic studies by Vinnik and co-workers (Vinnik and Montagner, 1996; Vinnik et al., 1997) show evidence for a weakly anisotropic (1.5\%) layer for S-waves at the bottom $40 \mathrm{~km}$ of the transition zone $(620-660 \mathrm{~km})$. The global tomography models (e.g. Montagner and Kennett, 1996; Montagner, 1998) also show significant transverse isotropic anisotropy in the transition zone with $\mathrm{V}_{\mathrm{SH}}>\mathrm{V}_{\mathrm{SV}}$ (Fig. 1) and $\mathrm{V}_{\mathrm{PH}}>$ VPV. Given the low intrinsic anisotropy of most of the minerals in the lower part of the transition zone, Karato (1998) suggested that this anisotropy is due to petrological layering caused by garnet and ringwoodite rich layers of transformed subducted oceanic crustal material. Such transversely isotropic medium with a vertical symmetry axis would not cause any splitting for vertically propagating $S$ waves.

The strongest seismic discontinuity at $660 \mathrm{~km}$ is due to the dissociation of ringwoodite to perovskite and magnesiowüstite with a shear wave impedance contrast of $9.9 \%$ (e.g. Shearer 1996). The $660 \mathrm{~km}$ discontinuity has an important topography with local depressions of up to $60 \mathrm{~km}$ from the global average in subduction zones (e.g. Flanagan and Shearer, 1998). From 660 to $1000 \mathrm{~km} \mathrm{a}$ weak anisotropy is observed in the top of the lower mantle with $\mathrm{V}_{\mathrm{SH}}<\mathrm{V}_{\mathrm{SV}}$ (Fig. 1) and $\mathrm{V}_{\mathrm{PH}}<\mathrm{VPV}$ (e.g. Montagner and Kennett, 1996; Montagner, 1998). Karato (1998) attributed the anisotropy to the crystal preferred orientation of perovskite and possibly magnesiowüstite caused by plastic deformation in the convective boundary layer at the top of the lower mantle. In this depth range, Kawakatsu and Niu (1994) have identified a flat seismic discontinuity at $920 \mathrm{~km}$ with $\mathrm{S}$ to $\mathrm{P}$ converted waves with a $\mathrm{S}$-wave velocity change of $2.4 \%$ in Tonga, Japan Sea and Flores Sea subduction zones. They suggested that this feature is thermodynamically controlled by some sort of phase transformation (orthorhombic to cubic perovskite ?) or alternatively, we may suggest it marks the bottom of the anisotropic boundary layer proposed by Montagner (1998) and Karato (1998). Kingma et al. (1995) and Karki et al. (1997) have suggested that the transformation of the highly anisotropic $\mathrm{SiO}_{2}$ polymorphs stishovite to $\mathrm{CaCl}_{2}$ structure at $50 \pm 3 \mathrm{GPa}$ at room temperature may be the possible explanation of reflectivity in the top of the lower mantle. However, according to Kingma et al. (1995) the transformation would take place at $60 \mathrm{GPa}$ at lower mantle temperatures in the range 2000 to $2500 \mathrm{~K}$, corresponding to depth of $1200-1500$ $\mathrm{km}$, that is several hundred $\mathrm{km}$ below the $920 \mathrm{~km}$ discontinuity. It is highly speculative to suggest that free silica is responsible for the $920 \mathrm{~km}$ discontinuity as a global feature as proposed by Kawakatsu and Niu (1994). Ringwood 
(1991) suggested that $10 \%$ stishovite would be present from 350 to $660 \mathrm{~km}$ in subducted oceanic crust and this would increase to about $16 \%$ at $730 \mathrm{~km}$. Hence, in the subduction zones studied by Kawakatsu and Niu (1994), it is quite possible that significant stishovite could be present to 1200 $\mathrm{km}$ and may be a contributing factor to the seismic anisotropy of the top of the lower mantle. From $1000 \mathrm{~km}$ to $2700 \mathrm{~km}$ the lower mantle is isotropic for body waves or free oscillations (e.g. Meade et al., 1995; Montagner and Kennett, 1996). Karato et al. (1995) have suggested by comparison with deformation experiments of analogue oxide perovskite that the seismically isotropic lower mantle is undergoing deformation by superplasticity or diffusive creep which does not produce a crystal preferred orientation. In the bottom of the lower mantle, the D" layer (100 to $300 \mathrm{~km}$ thick) appears to be transversely isotropic with a vertical symmetry axis characterized by $\mathrm{V}_{\mathrm{SH}}>\mathrm{V}_{\mathrm{SV}}$ (Fig. 1) (see Kendall and Silver, 1998 and Kendall this volume for further details).

In this paper, we review our current knowledge of the seismic anisotropy of the constituent minerals of the Earth's mantle and our ability to extrapolate these properties to mantle conditions of temperature and pressure (Fig. 1). The methods of calculating the anisotropic polycrystalline properties are briefly reviewed and applied to mantle samples coming from the top $100 \mathrm{~km}$ of the mantle.

\section{SINGLE CRYSTAL ANISOTROPIC SEISMIC PROPERTIES}

To understand the anisotropic seismic behavior of polyphase rocks in the Earth's mantle it is instructive to first consider the properties of the component single crystals. In this section, we will emphasize the anisotropy of individual minerals rather than the magnitude of velocity. The percentage anisotropy (A) is defined here as $A=200$ (Vmaximum - Vminimum) / (Vmaximum + Vminimum), where the maximum and minimum are found by exploring a hemisphere of all possible propagation directions. Note for $\mathrm{P}$-wave velocities the anisotropy is defined by the maximum and minimum velocities in two different propagation directions, for example the maximum $\mathrm{A}$ is given by the maximum and minimum $\mathrm{Vp}$ in a hemisphere, or for $\mathrm{Vp}$ in two specific directions such as the vertical and horizontal can be used. For S-waves in an anisotropic medium there are two orthogonally polarized S-waves with different velocities for each propagation direction, hence A can be defined for each direction. The consideration of the single crystal properties is particularly important for the transition zone $(410-660 \mathrm{~km})$ and lower mantle (below $660 \mathrm{~km}$ ) as the deformation mechanisms and resulting preferred orientation of these minerals under the extreme conditions of temperature and pressure are very poorly documented by experimental investigations. In compiling Table 1 of anisotropic single crystal properties we have chosen, where possible, to include the most recent experimental determinations, $50 \%$ of the data has been published in the last nine years and over $20 \%$ has been published in the last two years. A major trend in recent years is the use of computational modeling to determine the elastic constants at very high pressures. The theoretical modeling gives a first estimate of the pressure derivatives in a pressure range not currently accessible to direct measurement (e.g. Karki et al., 1997). Although there is an increasing amount of single crystal data available to high temperature or high pressure, no data is available for simultaneous high temperature and pressure of the Earth's mantle (See Fig.1 for the pressure and temperatures).

\subsection{Upper Mantle}

The upper mantle (down to $410 \mathrm{~km}$ ) is composed of three anisotropic and volumetrically important phases (Fig. 2, top); olivine, enstatite (orthopyroxene) and diopside (clinopyroxene). The seismic properties of these single crystal are presented Fig. 2, top. The other volumetrically important phase is garnet, which is nearly isotropic and hence not of great significance to our discussion.

Olivine - A certain number of accurate determinations of the elastic constants of olivine are now available which all agree that the anisotropy of $\mathrm{Vp}$ is $25 \%$ and maximum anisotropy of $\mathrm{Vs}$ is $18 \%$ at ambient conditions for a mantle composition of about Fo90. The first order temperature derivatives have been determined between 295-1500K (Issak, 1992). The first and second order pressure derivatives for olivine were first determined to $3 \mathrm{GPa}$ by Webb (1989). However, a more recent determination to $17 \mathrm{GPa}$ by Abramson et al. (1997) has shown that the second order derivative is only necessary for elastic stiffness modulus $\mathrm{C}_{55}$. The first order derivatives are in good agreement between these two studies. The anisotropy of the olivine single crystal increases slightly with temperature (+2\%) using the data of Issak (1992) and reduces slightly with increasing pressure using the data of Abramson et al. (1997). One would expect the single crystal anisotropy to be nearly constant along a geotherm.

Orthopyroxene - The elastic properties of orthopyroxene (Enstatite or Bronzite) with a magnesium number $(\mathrm{Mg} / \mathrm{Mg}+\mathrm{Fe})$ near the typical upper mantle value of 0.9 has also been extensively studied. The $\mathrm{Vp}$ anisotropy varies between 15.1\% (En80 Bronzite; Frisillo and Barsch, 1972) and 12.0\% (En100 Enstatite; Weidner et al., 1978) and the maximum Vs anisotropy between 15.1\% (En80 Bronzite; Webb and Jackson, 1993) and 11.0\% (En100 Enstatite; Weidner et al., 1978). Some of the variation in the elastic constants and anisotropy may be related to composition and structure in the orthopyroxenes (Duffy and Vaughan, 1989). The first order temperature derivatives have been determined over a limited range between 298-623K (Frisillo and Barsch, 1972). The first and second order pressure derivatives for Enstatite have been recently determined up to $12.5 \mathrm{GPa}$ by Chai et al. (1997b). This study confirms an earlier one of Webb and Jackson to $3 \mathrm{GPa}$ which showed that first and 


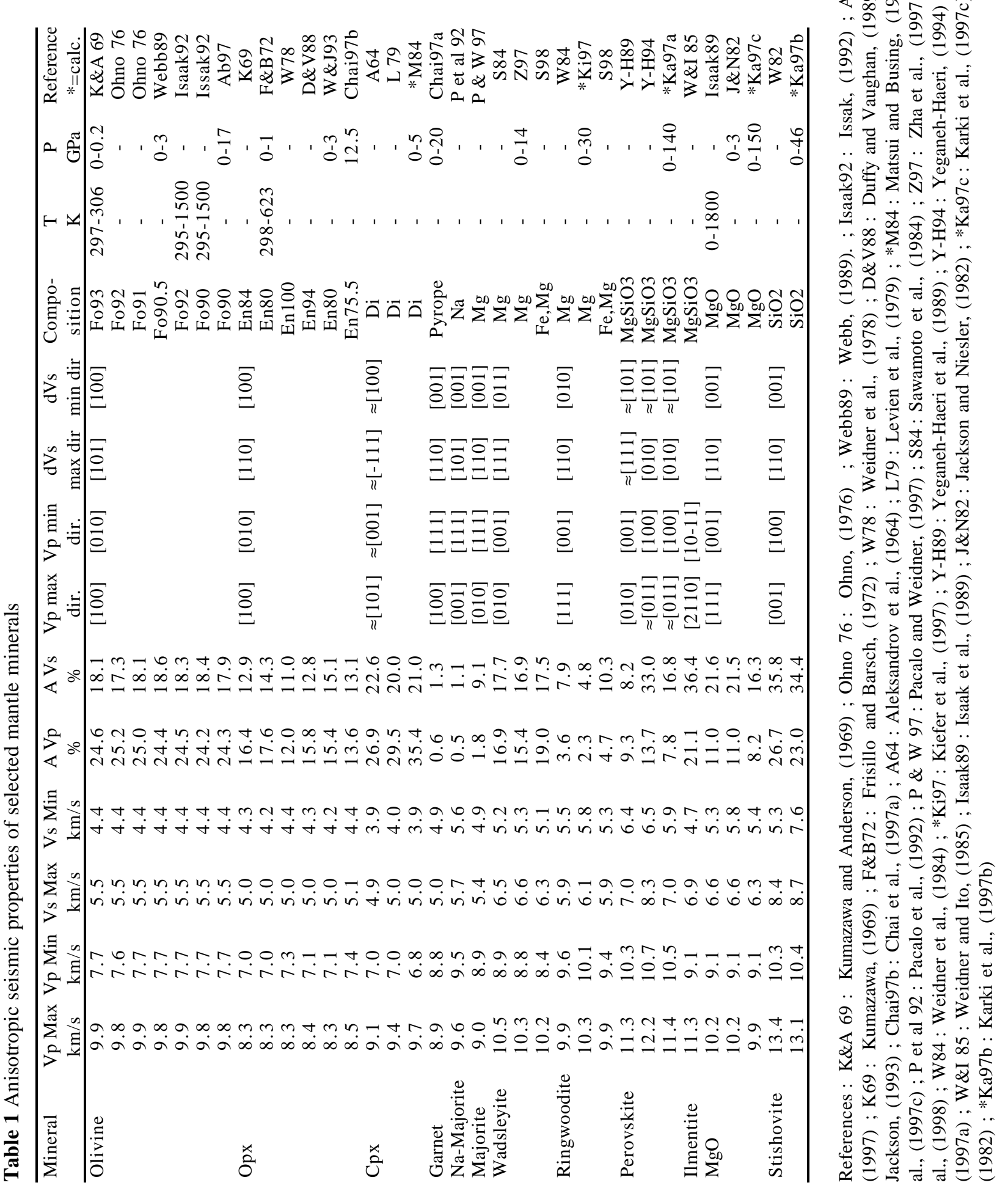

๘ं

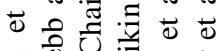

范...

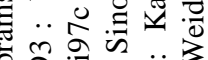

娄宁 ...

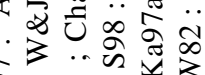

……

《ล̊응ㅎㅇ

$\widehat{\tau}=0 \hat{己} \partial$

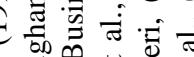

元氙

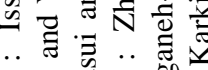

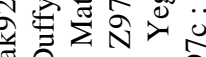

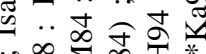

$>^{*}=\lambda$

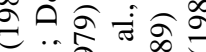

요웡

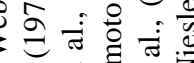

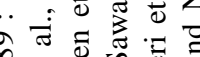

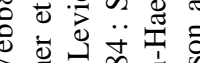

훙 $\cdots$

…요잉

$\infty \overparen{f}=\cdots$

$\beta \varrho \overrightarrow{0})^{\infty}$

สิ่

.

잏

颉 䒕

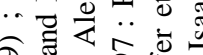
인

.

ส…ำ.

종ํ..

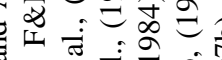

$\cdots$ 元

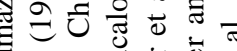

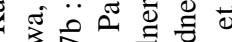

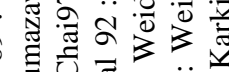

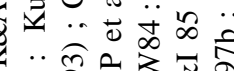

.

드응

ฯ 


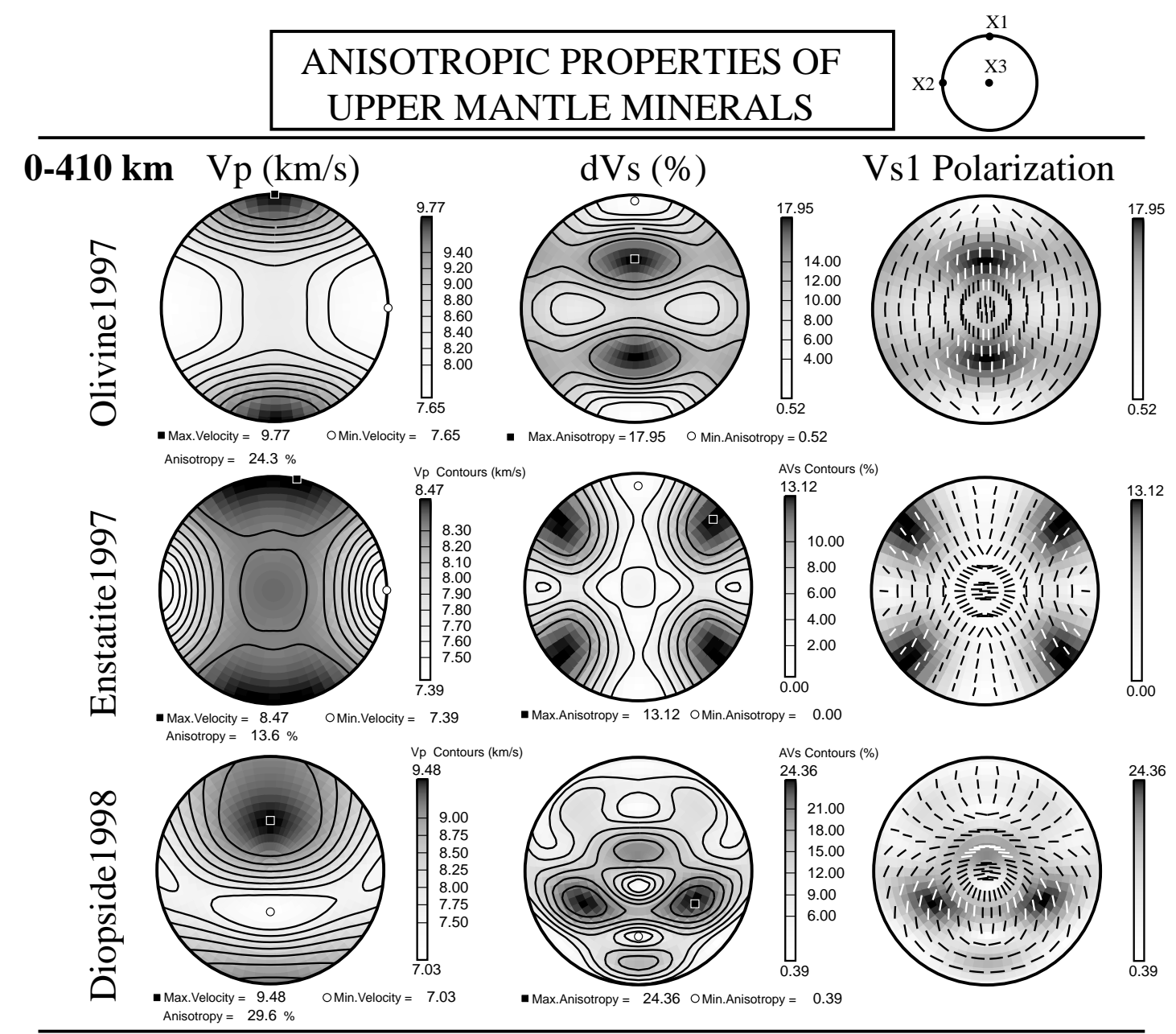

\section{0-660 km, transition zone}
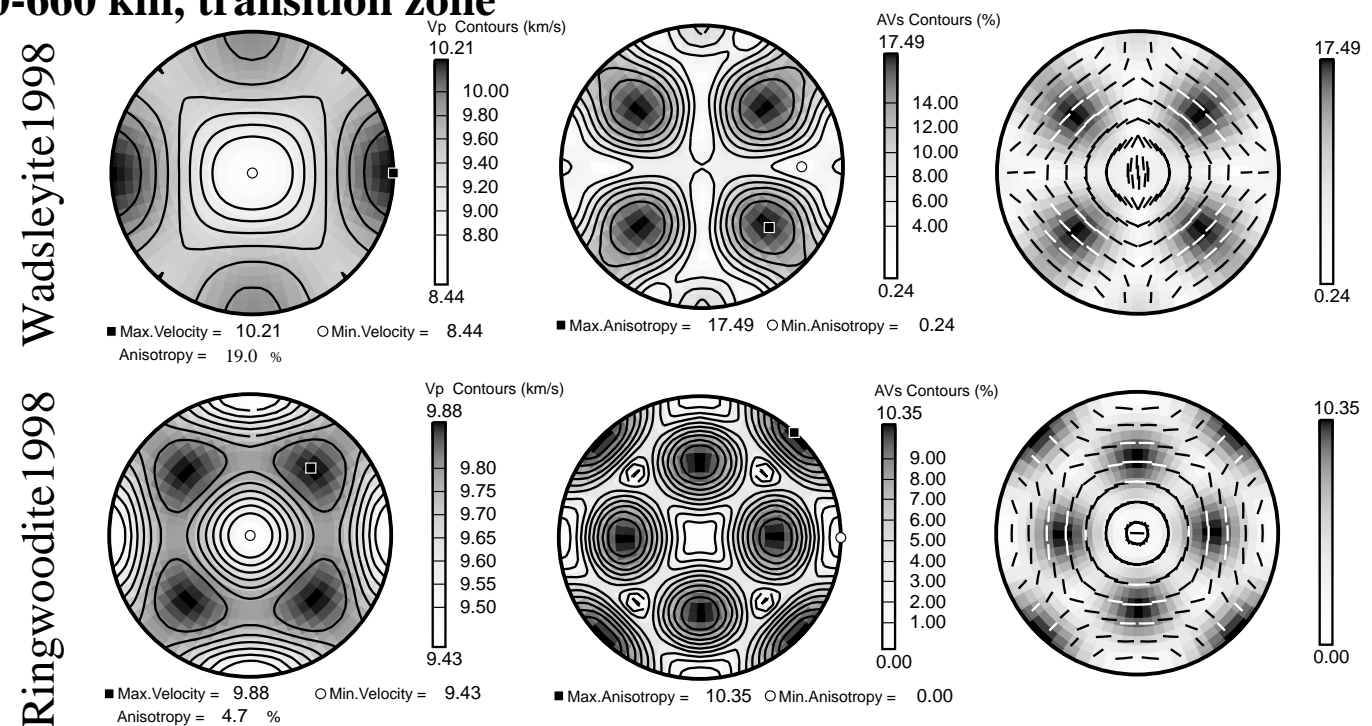

Figure 2. The seismic anisotropy of single crystals of upper mantle minerals. Projections are upper hemisphere. All properties calculated at room pressure and temperature. X1, X2, X3 are the reference axes for the elastic constants, $X 1=[100], X 2=[010]$ and $X 3=[001]$ for cubic, tetragonal and orthorhombic crystals. $\mathrm{X} 1=\perp(100) \mathrm{X} 2=[010]$ and $\mathrm{X} 3=[001]$ for monoclinic. Contours in $\mathrm{km} / \mathrm{s}$ for $\mathrm{Vp}$ (left) and in \% anisotropy for dVs (middle). Right: trace of the Vs1 polarization plane. 
second order pressure derivatives are needed to describe the elastic constants at mantle pressures. The anisotropy of $\mathrm{Vp}$ and Vs does not vary significantly with pressure using the data of Chai et al. (1997b) to $12.5 \mathrm{GPa}$. The anisotropy of $\mathrm{Vp}$ and $\mathrm{Vs}$ does increase by about $3 \%$ when extrapolating to $1000^{\circ} \mathrm{C}$ using the first order temperature derivatives of Frisillo and Barsch (1972).

Clinopyroxene - The elastic constants of clinopyroxene (Diopside) of mantle composition has only been experimentally measured at ambient conditions (Levien et al., 1979; Collins and Brown 1998), both studies show that $\mathrm{Vp}$ anisotropy is $29 \%$ and $\mathrm{Vs}$ anisotropy is between 20 to $24 \%$. There are no measured single crystal pressure derivatives. In one of the first calculations of the elastic constants of a complex silicate at high pressure, Matsui and Busing (1984) predicted the first order pressure derivatives of diopside from 0 to $5 \mathrm{GPa}$. The calculated elastic constants at ambient conditions are in good agreement with the experimental values and the predicted anisotropy for $\mathrm{Vp}$ and Vs of $35.4 \%$ and $21.0 \%$ respectively is also in reasonable agreement. The predicted bulk modulus of $105 \mathrm{GPa}$ is close to the experimental value of $108 \mathrm{GPa}$ given by Levien et al. (1979). The pressure derivative of the bulk modulus 6.2 is slightly lower than the value of $7.8 \pm 0.6$ given by Bass et al. (1981). Using the elastic constants of Matsui and Busing (1984) the $\mathrm{Vp}$ anisotropy decreases from $35.4 \%$ to $27.7 \%$ and Vs increases from $21.0 \%$ to $25.5 \%$ with increasing pressure from ambient to $5 \mathrm{GPa}$. To allow calculations of seismic properties at mantle pressures, Estey and Douglas (1986) have proposed using $80 \%$ of the orthopyroxene pressure derivatives of Frisillo and Barsch (1972). However, comparison of the most recent orthopyroxene pressure derivatives of Chai et al. (1997b) with the calculated values for clinopyroxene of Matsui and Busing (1984) shows that they differ by $200 \%$ for $\mathrm{C}_{11}, \mathrm{C}_{33}$ and $\mathrm{C}_{44}$ suggesting large errors would occur in using the method proposed by Estey and Douglas (1986). We would recommend using the values given by Matsui and Busing (1984) until an experimental determination has been made. A major problem still remains as no clinopyroxene temperature derivatives are available. Estey and Douglas (1986) proposed using the orthopyroxene temperature derivatives of Frisillo and Barsch (1972). For completeness garnet has been included in Table 1. Garnet is nearly isotropic with $\mathrm{Vp}$ anisotropy of $0.6 \%$ and $\mathrm{Vs}$ of $1.3 \%$.

\subsection{Transition Zone}

Over the last 25 years a major effort has been made to experimentally determine the phase petrology of the transition zone and lower mantle. Whereas single crystals of upper mantle phases are readily available, single crystals of transition zone and lower mantle for elastic constant determination have to be grown at high pressure and high temperature. The petrology of the transition zone is dominated by garnet, majorite, wadsleyite, ringwoodite, calcium rich perovskite, clinopyroxene and possibly stishovite. The seismic anisotropy of wadsleyite and ringwoodite are illustrated in Fig. 2, bottom.

Majorite - The pure $\mathrm{Mg}$ end member majorite of the majorite-pyrope garnet solid solution has tetragonal symmetry and is weakly anisotropic with $1.8 \%$ for $\mathrm{Vp}$ and $9.1 \%$ for Vs (Pacalo and Weidner, 1997). A recent study of the majorite - pyrope system by Heinemann et al. (1997) shows that tetragonal form of majorite is restricted to a composition of less $20 \%$ pyrope and hence is unlikely to exist in the Earth's transition zone. Majorite with cubic symmetry is nearly isotropic with $\mathrm{Vp}$ anisotropy of $0.5 \%$ and Vs of $1.1 \%$. No pressure or temperature derivatives have been determined for tetragonal or cubic majorite. Cubic majorite has very similar properties to pyrope garnet (Chai et al., 1997a) as might be expected.

Wadsleyite - The elastic constants of $\mathrm{Mg}_{2} \mathrm{SiO}_{4}$ wadsleyite were first determined by Sawamoto et al. (1984) and this early determination was confirmed by Zha et al. (1997) with a Vp anisotropy of $16 \%$ and Vs of $17 \%$. The $(\mathrm{Mg}, \mathrm{Fe})_{2} \mathrm{SiO}_{4}$ wadsleyite has slightly lower velocities and higher anisotropies (Sinogeikin et al., 1998). The first order pressure derivatives determined from the data of Zha et al. (1997) to $14 \mathrm{GPa}$ show the anisotropy of $\mathrm{Mg}_{2} \mathrm{SiO}_{4}$ wadsleyite decreases slightly with increasing pressure. At pressures corresponding to the $410 \mathrm{~km}$ seismic discontinuity (ca $13.8 \mathrm{GPa}$ ), the $\mathrm{Vp}$ anisotropy would be $11.0 \%$ and $\mathrm{Vs}$ $12.5 \%$.

Ringwoodite - The elastic constants of $\mathrm{Mg}_{2} \mathrm{SiO}_{4}$ ringwoodite were first measured by Weidner et al. (1984) and $(\mathrm{Mg}, \mathrm{Fe})_{2} \mathrm{SiO}_{4}$ ringwoodite by Sinogeikin et al. (1998) at ambient conditions with $\mathrm{Vp}$ anisotropy of 3.6 and $4.7 \%$, and Vs of 7.9 and $10.3 \%$ respectively. Kiefer et al. (1997) have calculated the elastic constants of $\mathrm{Mg}_{2} \mathrm{SiO}_{4}$ ringwoodite to $30 \mathrm{GPa}$. Their constants at ambient conditions gives a $\mathrm{Vp}$ anisotropy of $2.3 \%$ and Vs of $4.8 \%$ very similar to the experimental results of Weidner et al. (1984). There is a significant variation (5\% to $0 \%$ ) of the anisotropy of ringwoodite with pressure, $15 \mathrm{GPa}$ (ca $500 \mathrm{~km}$ depth) the $\mathrm{Vp}$ anisotropy is $0.4 \%$ and $\mathrm{Vs}$ is $0.8 \%$, hence ringwoodite is nearly perfectly isotropic at transition zone pressures. No single crystal temperature derivatives have been measured for wadsleyite or ringwoodite. Olivine transforms to wadsleyite at about $410 \mathrm{~km}$, and wadsleyite transforms to ringwoodite at about $500 \mathrm{~km}$, both transformations result in a decrease in anisotropy with depth. The gradual transformation of clinopyroxene to majorite between 400 and $475 \mathrm{~km}$ would also result in a decrease in anisotropy with depth.

\subsection{Lower Mantle}

The lower mantle is essentially composed of perovskite, magnesiowüstite and possibly minor amount of $\mathrm{SiO}_{2}$ in the form of stishovite in the top part of the lower mantle (e.g. Ringwood, 1991). $\mathrm{MgSiO}_{3}$ may be in the form of perovskite or possibly ilmenite. The ilmenite structured 
$\mathrm{MgSiO}_{3}$ is most likely to occur at bottom of the transition zone and top of the lower mantle. Seismic properties of the main constituent mineral of the lower mantle are presented Fig. 3.

Perovskite $\left(\mathrm{MgSiO}_{3}\right)$ - The first determination of the elastic constants of $\mathrm{MgSiO}_{3}$ perovskite at ambient conditions was given by Yeganeh-Haeri et al. (1989). However this determination has been replaced by a more accurate study of a better quality crystal (Yeganeh-Haeri, 1994). The 1989 and 1994 studies give completely different velocity distributions and hence very different anisotropies. The 1989 and 1994 studies gives Vp anisotropy of $9.3 \%$ and $13.7 \%$ respectively and Vs of $8.2 \%$ and $33.0 \%$ respectively. More importantly the [010] direction is minimum for Vs anisotropy in the 1989 determination and the maximum Vs anisotropy for 1994. Karki et al. (1997a) calculated the elastic constants of $\mathrm{MgSiO}_{3}$ perovskite $140 \mathrm{GPa}$. The calculated constants are in close agreement with the experimental measurements of Yeganeh-Haeri (1994). Karki et al (1997a) found that significant variations in anisotropy occurred with increasing pressure, first decreasing to $6 \%$ at $20 \mathrm{GPa}$ for $\mathrm{Vp}$ and to $8 \%$ at $40 \mathrm{GPa}$ for Vs and then increasing to $12 \%$ and $16 \%$ respectively at $140 \mathrm{GPa}$. At the $660 \mathrm{~km}$ seismic discontinuity (ca $23 \mathrm{GPa}$ ) the Vp and Vs anisotropy would be $6.5 \%$ and $12.5 \%$ respectively. The $\mathrm{MgSiO}_{3}$ ilmenite of trigonal symmetry is very anisotropic at ambient conditions (Weidner and Ito, 1985; Da Silva et al., 1999) with Vp anisotropy of $21.1 \%$ and Vs of $36.4 \%$.

Magnesiowüstite - The other major phase is magnesiowüstite $(\mathrm{Mg}, \mathrm{Fe}) \mathrm{O}$, for which the elastic constants have not been determined. The elastic constants of the pure end member periclase $\mathrm{MgO}$ of the $\mathrm{MgO}-\mathrm{FeO}$ solid solution series has been measured to $3 \mathrm{GPa}$ by Jackson and Niesler (1982). The temperature derivatives for $\mathrm{MgO}$ have been measured by Isaak et al. (1989) to $1800 \mathrm{~K}$. Both these studies indicate a Vp anisotropy of $11.0 \%$ and $\mathrm{Vs}$ of $21.5 \%$ at ambient conditions. Karki et al. (1997c) calculated the elastic constants of $\mathrm{MgO}$ to $150 \mathrm{GPa}$. They find considerable changes in anisotropy with increasing pressure, first decreasing to $2 \%$ for $\mathrm{Vp}$ and $4 \%$ for Vs at $10 \mathrm{GPa}$ and then increasing to $22 \%$ and $55 \%$ respectively at $150 \mathrm{GPa}$. The anisotropy of $\mathrm{MgO}$ increases linearly from $11.0 \%$ and $21.5 \%$ for $\mathrm{Vp}$ and $\mathrm{Vs}$ respectively at ambient conditions to $20 \%$ and $42 \%$ respectively at $1800 \mathrm{~K}$ according to the data of Isaak et al. (1989). The anisotropy of Vp and Vs at the $660 \mathrm{~km}$ (ca $23 \mathrm{GPa}$ and $2000 \mathrm{~K}$ ) is predicted to be $4 \%$ and $6 \%$ according to calculated constants of Karki et al (1997c) at ambient temperature and $23 \mathrm{GPa}$, whereas the measured constants of Isaak et al. (1989) at $1800 \mathrm{~K}$ and ambient pressure have much higher anisotropies. This case illustrates the major problem for other minerals where we lack temperature derivatives. Furthermore not only the magnitude of the anisotropy of $\mathrm{MgO}$, but also the orientation of the anisotropy changes with increasing pressure according to the calculations of Karki et al. (1997c), for example the fastest $\mathrm{Vp}$ is parallel to
[111] at ambient pressure and becomes parallel to [100] at $150 \mathrm{GPa}$ pressure.

$\mathrm{SiO}_{2}$ Polymorphs - Finally, the free $\mathrm{SiO}_{2}$ in the transition zone and the top of the lower mantle (to a depth of $1180 \mathrm{~km}$ or $47 \mathrm{GPa}$ ) will be in the form of stishovite. The original experimental determination of the single crystal elastic constants of stishovite by Weidner et al. (1982) and the more recent calculated constants of Karki et al. (1997b) both indicate a $\mathrm{Vp}$ and $\mathrm{Vs}$ anisotropy at ambient conditions of 26.7 to $23.0 \%$ and 35.8 to $34.4 \%$ respectively, making this a highly anisotropic phase. The calculations of Karki et al (1997b) show that the anisotropy increases dramatically as the phase transition to $\mathrm{CaCl}_{2}$ structured $\mathrm{SiO}_{2}$ is approached at $47 \mathrm{GPa}$. The $\mathrm{Vp}$ anisotropy increases from $23.0 \%$ to $28.9 \%$ and $\mathrm{Vs}$ from $34.4 \%$ to $161.0 \%$ with increasing pressure from ambient to $47 \mathrm{GPa}$. The maximum $\mathrm{Vp}$ is parallel to [001] and the minimum parallel to [100]. The maximum dVs is parallel to [110] and the minimum parallel to [001].

In conclusion, we can say that the general trend favors an anisotropy decrease with increasing pressure and increase with increasing temperature, olivine is a good example of this behavior. The changes are limited to a few percent in most cases. The primary causes of the anisotropy changes are minor crystal structural rearrangements rather than velocity changes due to density change caused by compressibility with pressure or thermal expansion with temperature. The effect of temperature is almost perfectly linear in almost all cases, some minor non-linear effects are seen in $\mathrm{MgO}$. Non-linear effects with increasing pressure on the elastic constants causes the anisotropy of Wadsleyite, Ringwoodite, Perovskite and $\mathrm{MgO}$ to first decrease until a minimum value is reached and then increase. In the case of $\mathrm{MgO}$, the changes with pressure are dramatic. Stishovite also shows major changes in anisotropy in the pressure range close to the transformation to the $\mathrm{CaCl}_{2}$ structure. The single crystal temperature derivatives of clinopyroxene, wadsleyite, ringwoodite, perovskite and stishovite are currently unknown which make quantitative seismic anisotropic modeling of the transition zone and lower mantle speculative. To illustrate the variation of anisotropy as a function of mantle conditions of temperature and pressure, we have calculated the seismic properties along a mantle geotherm (Fig. 4a,b). The mantle geotherm is based on the PREM model for the pressure scale. The temperature scale is based on the continental geotherm of Mercier (1980) from the surface to $130 \mathrm{~km}$ and Ito and Katsura (1989) for the transition zone and Brown and Shankland (1981) for the lower mantle. The upper mantle minerals olivine (Vp, Vs) and enstatite $(\mathrm{Vp})$ show a slight increase of anisotropy in the first $100 \mathrm{~km}$ due to the effect of temperature (Fig. 4a). With increasing depth, the trend is for decreasing anisotropy except for Vs of enstatite and diopside. In the transition zone and lower mantle, the situation is more complex due to the 


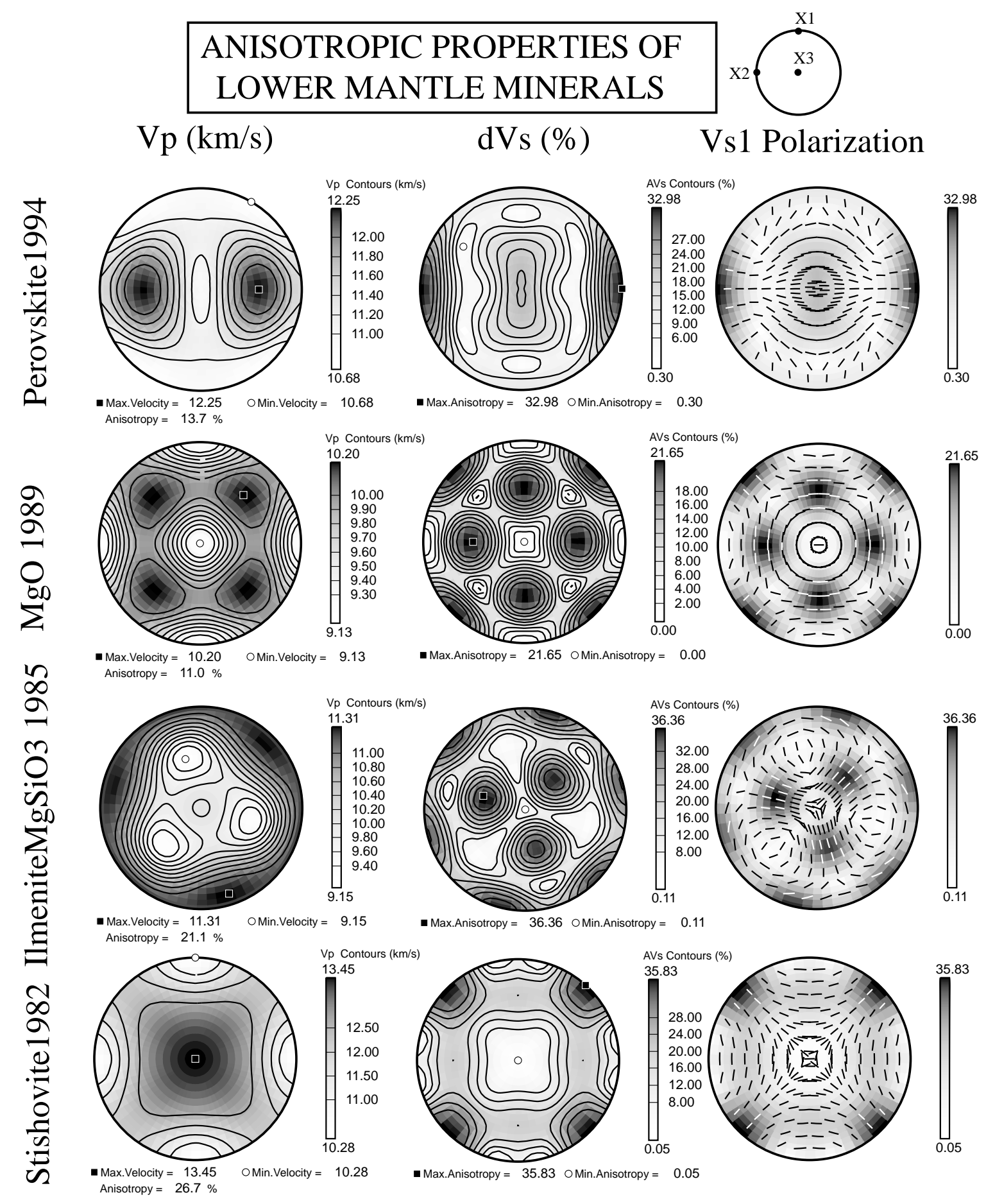

Figure 3. The seismic anisotropy of single crystals of lower mantle minerals. Projections are upper hemisphere. All properties calculated at room pressure and temperature. X1, X2,X3 are the reference axes for the elastic constants, $\mathrm{X} 1=[100], \mathrm{X} 2=[010]$ and $\mathrm{X} 3=[001]$ for cubic, tetragonal and orthorhombic crystals. $\mathrm{X} 1=[\mathrm{a}]$ $\mathrm{X} 2=[\mathrm{m}]$ and $\mathrm{X} 3=[\mathrm{c}]$ for trigonal. Contours in $\mathrm{km} / \mathrm{s}$ for Vp (left) and in \% anisotropy for dVs (middle). Right: trace of the Vs1 polarization plane. 

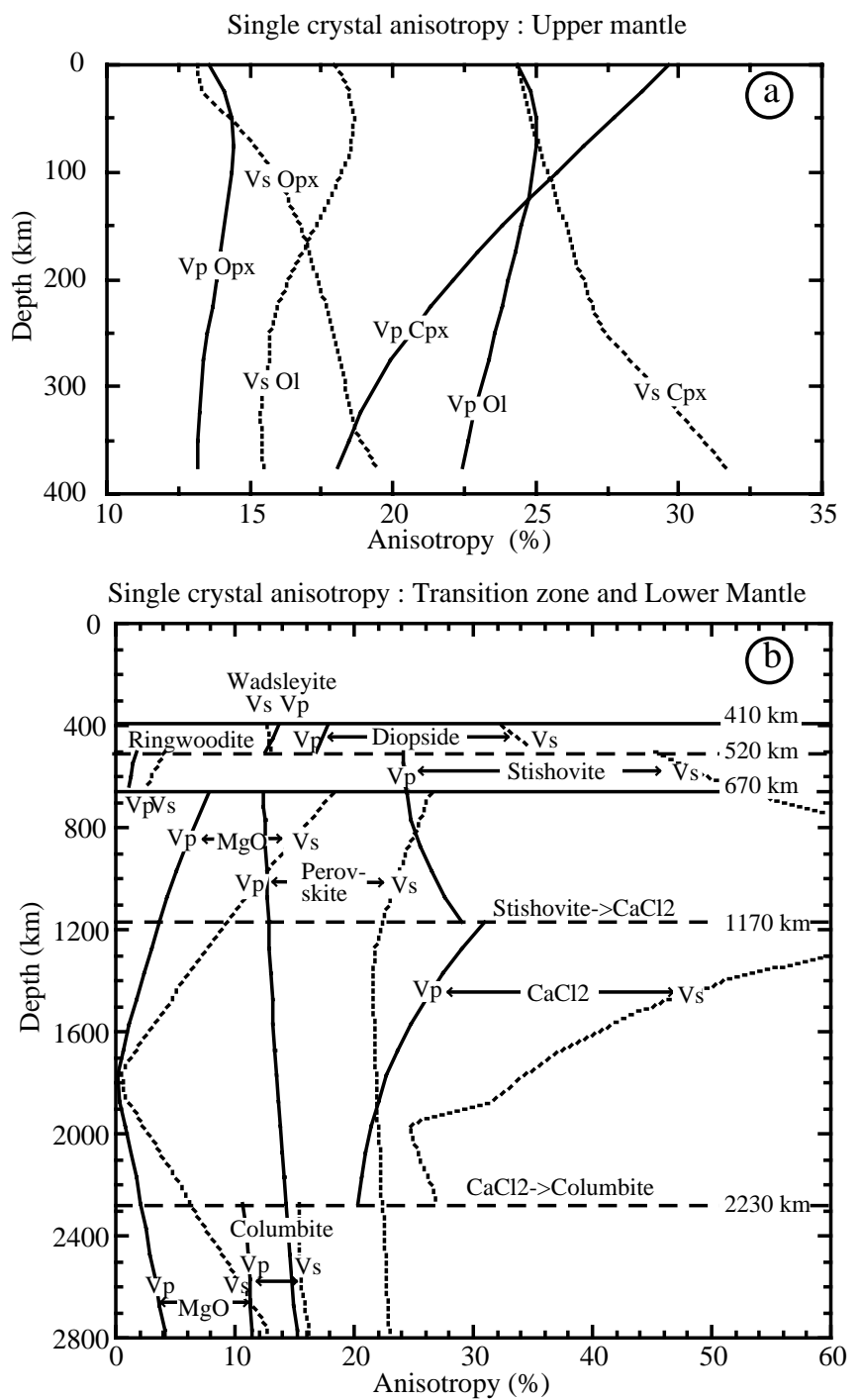

Figure 4. a) The maximum seismic anisotropy of single crystals of upper mantle minerals as a function of depth along a mantle geotherm (see text for details). b) The maximum seismic anisotropy of single crystals of lower mantle minerals as a function of depth along a mantle geotherm (see text for details).

presence of phase transitions. In the transition zone (Fig. 4b) diopside may be present to about $500 \mathrm{~km}$ with an increasing $\mathrm{Vs}$ and decreasing $\mathrm{Vp}$ anisotropy with depth. Wadsleyite is less anisotropic than olivine at $410 \mathrm{~km}$, but significantly more anisotropic than ringwoodite found below $520 \mathrm{~km}$. Although the lower mantle is known to be seismically isotropic, the constituent minerals are anisotropic. $\mathrm{MgO}$ shows important variations in anisotropy with depth : it is anisotropic at $670 \mathrm{~km}$ and $2800 \mathrm{~km}$, with different directions of anisotropy, but isotropic at about 1800 $\mathrm{km}$. Perovskite is strongly anisotropic throughout the lower mantle. The $\mathrm{SiO}_{2}$ polymorphs are all strongly anisotropic, particularly for $\mathrm{S}$ waves. If free silica is present in the transition zone or lower mantle, due perhaps to the presence of subducted basalt (e.g. Ringwood, 1991), then even a small volume fraction of the $\mathrm{SiO}_{2}$ polymorphs could influence the seismic anisotropy of the mantle. However, to do so, the $\mathrm{SiO}_{2}$ polymorphs would have to be oriented, either due to dislocation glide (plastic flow), oriented grain growth or anisometric crystal shape (viscous flow) (e.g. Mainprice and Nicolas, 1989). Given that the $\mathrm{SiO}_{2}$ polymorphs are likely to be less $10 \%$ by volume (Ringwood, 1991) and hence would not be the load bearing framework of the rock, it is more likely that the inequant shape of $\mathrm{SiO}_{2}$ polymorphs would control their orientation during viscous flow.

The pyrolite and piclogite models of mantle petrology provide a framework for testing the mineral physics against global seismic models (e.g. Weidner, 1985, 1986; Montagner and Anderson, 1989; Ita and Stixrude, 1992). The pyrolite and piclogite models of Ita and Stixrude (1992) are shown in Fig. 5 where we have grouped together the structurally similar isotropic phases garnet and majorite. The difference in mineralogy of these models will result in a difference of magnitude and orientation of anisotropy as a function of depth. The volumetrically important phases will dominate the seismic anisotropy, especially if they have a moderate to strong anisotropy. The exact depth range of orthopyroxene may be less than proposed by Ita and Stixrude (1992) as several experimental studies (Kanzaki, 1991; Pacalo and Gasparik, 1990; Woodland and Angel, 1997) show that orthopyroxene transforms to clinopyroxene at about $300 \mathrm{~km}$. To illustrate the effect of magnitude and orientation of anisotropy, we have plotted Fig. 6 the seismic parameter $\Phi=\left(\mathrm{V}_{\mathrm{PV}} / \mathrm{V}_{\mathrm{PH}}\right)^{2}$ (e.g. Montagner and Anderson, 1989) where $\mathrm{V}_{\mathrm{PV}}$ is the vertical and $\mathrm{V}_{\mathrm{PH}}$ is horizontal seismic $\mathrm{P}$ wave velocity. An isotropic medium has a $\Phi=1$, when $\mathrm{V}_{\mathrm{PV}}<\mathrm{V}_{\mathrm{PH}}$ then $\Phi<1$ and $\mathrm{V}_{\mathrm{PV}}>\mathrm{V}_{\mathrm{PH}}$ then $\Phi>1$. We have chosen a $\mathrm{P}$-wave parameter because the body-wave and normal mode reference earth models of Montagner and Kennett (1996) are more robust for P-waves than S-waves. The robustness can be illustrated by the fact that P-wave model is less sensitive to the number of seismic parameters used in the inversion procedure than the S-wave model (see Montagner and Kennett 1996, their figures 8 and 12). In the case of the single crystals, we have placed the reported dislocation glide direction parallel to horizontal and the pole to glide plane parallel to vertical as for horizontal simple shear flow (Fig. 6a). This approach is a great simplification from the case of polycrystals with LPO, especially in higher symmetry crystals, such as cubic. We can compare this directly to various seismic models Fig. 6b (Montagner and Kennett, 1996; Montagner, 1998). The values of $\Phi$ are much larger for the single crystals than for the global seismic models as is to be expected, however some interesting trends are observed. In the $0-410 \mathrm{~km}$ depth range were olivine is the volumetrically dominant phase in both the pyrolite and piclogite models (Fig. 5) olivine has a $\Phi$ value of much less than 1.0. The second most important 


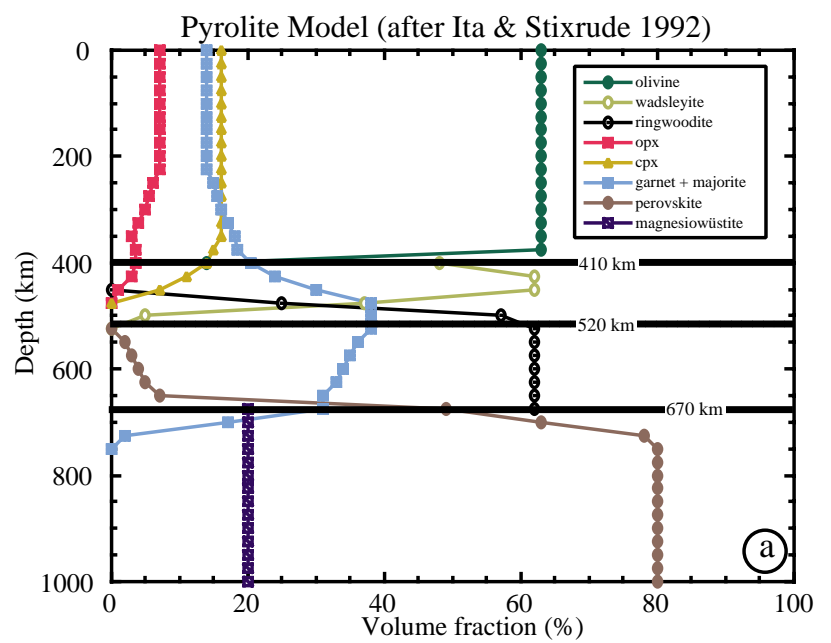

Piclogite Model (after Ita \& Stixrude 1992)

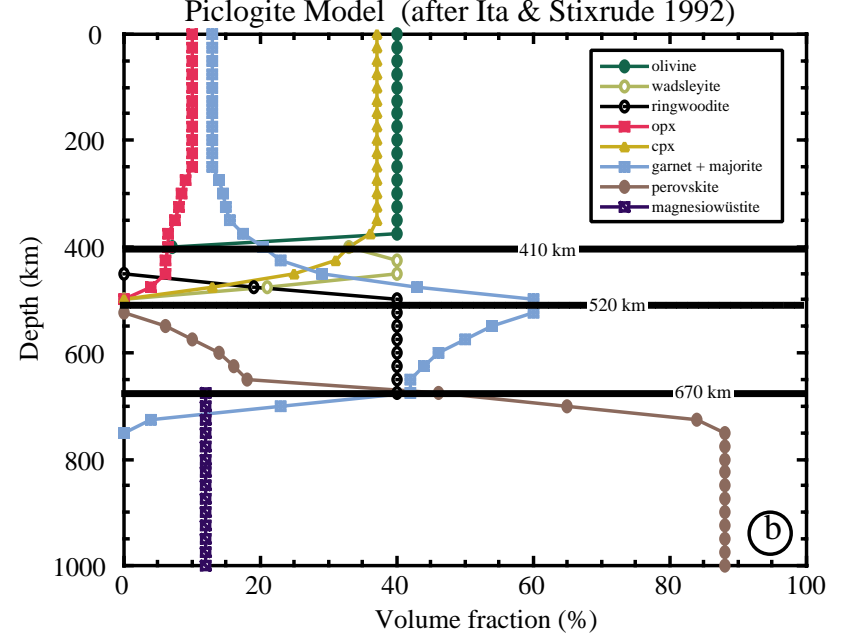

Figure 5. a) The pyrolite model of Ita \& Stixrude (1992) presented as volume fractions. b) The piclogite model of Ita \& Stixrude (1992). The nearly isotropic minerals garnet and majorite have summed together as the cubic majorite has very similar elastic properties. The $\mathrm{Ca}$ and $\mathrm{Mg}$ rich perovskites have also been summed together as not single crystal constants for $\mathrm{Ca}$ perovskite are currently available.

phase in both models is clinopyroxene and this also has a $\Phi$ value of less than 1.0, hence we would expect a $\Phi$ less than 1.0 for the $0-410 \mathrm{~km}$ depth range, which is observed in the seismic models (Fig. 6b). In the transition zone both wadsleyite and ringwoodite have a $\Phi$ value of more than 1.0 whereas the seismic models all have a value of less than one. In this case, it would appear that the observed seismic anisotropy cannot be explained by plasticity of wadsleyite and ringwoodite and another explanation has to be sort, such as layering (Karato, 1998). In the lower mantle we have only plotted data for $\mathrm{MgO}$ and perovskite as the glide system for these minerals is well established. For the top of the lower mantle, both $\mathrm{MgO}$ and perovskite have a $\Phi$ value greater
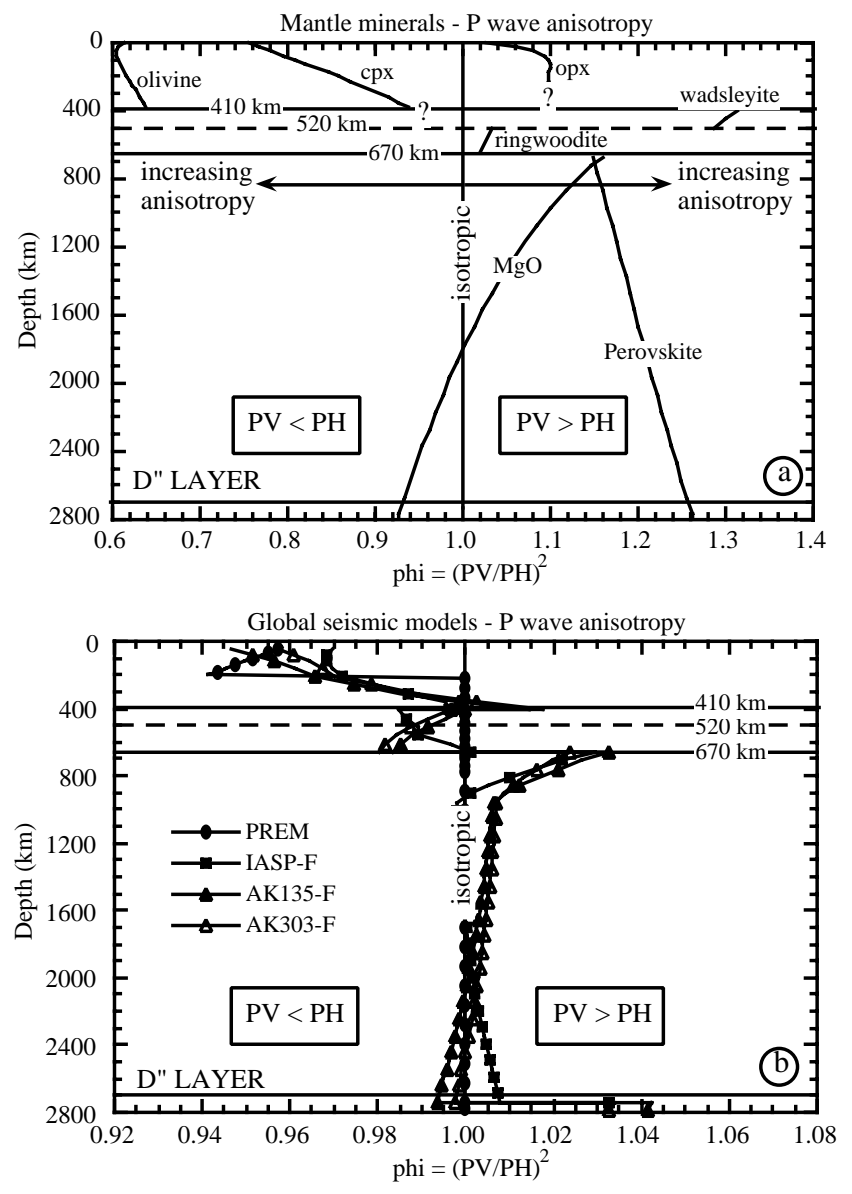

Figure 6. a) The $\mathrm{P}$ wave anisotropy parameter $\Phi=$ $\left(\mathrm{V}_{\mathrm{PV}} / \mathrm{V}_{\mathrm{PH}}\right)^{2}$ as a function of depth for mantle minerals assuming that the glide direction is horizontal and normal to the glide plane is vertical as would be the case for horizontal flow. b) The $\mathrm{P}$ wave anisotropy parameter $\Phi=\left(\mathrm{V}_{\mathrm{PV}} / \mathrm{V}_{\mathrm{PH}}\right)^{2}$ as a function of depth for global seismic models (Montagner and Kennett, 1996). Note horizontal scale is different in figures a and $\mathrm{b} .(\mathrm{PH}=\mathrm{Vp}$ horizontal, $\mathrm{PV}=\mathrm{Vp}$ vertical $)$

than 1.0. In the same region, the seismic models also have $\Phi$ value of greater than 1.0 suggesting that plasticity could be responsible for the observed anisotropy in this depth range (Karato, 1998). However, for most of the lower mantle the observed $\Phi$ value is close to 1.0 suggesting that the lower mantle is isotropic (Fig. 6b). In the D" layer at the bottom of the lower mantle $\Phi$ is again greater than 1.0 with a value similar to the $670 \mathrm{~km}$ discontinuity. From our plot of perovskite (Fig. 6a), $\Phi$ is also greater than 1.0 in the D" layer, so perhaps due to the plasticity of constituent minerals (such as perovskite). Note this is in contradiction to the interpretation as a layered structure (e.g. Kendall and Silver, 1998) based on regional observations of $\mathrm{V}_{\mathrm{SH}}>\mathrm{V}_{\mathrm{SV}}$. The $\mathrm{V}_{\mathrm{PV}}>\mathrm{V}_{\mathrm{PH}}$ and $\mathrm{V}_{\mathrm{SH}}>\mathrm{V}_{\mathrm{SV}}$ in the $\mathrm{D}$ " layer from the global models of Montagner and Kennett (1996) cannot be 
explained by a layered structure. However this does not preclude that locally, for example below subduction regions, the D" layer may be layered.

\section{ESTIMATION OF ANISOTROPIC POLYCRYSTALLINE PHYSICAL PROPERTIES}

The calculation of the physical properties from microstructural information (crystal orientation, volume fraction, grain shape etc.) is important for mantle rocks because it gives insight into the role of microstructure in determining the bulk properties. A calculation can be made for the in-situ state at high temperature and pressure of the mantle for samples where the microstructure has been changed by subsequent chemical alteration (e.g. the transformation olivine to serpentine) or mechanically induced changes (e.g. fractures created by decompression). The in-situ mantle temperatures and pressures can be simulated using the appropriate single crystal derivatives (see below). Additional features not necessarily preserved in the recovered microstructure, such as the presence of fluids (e.g. magma) can be modeled (e.g. Blackman and Kendall, 1997; Mainprice 1997; Williams and Garnero, 1996). Finally, the effect of phase change on the physical properties can also be modeled using these methods (e.g. Mainprice et al., 1990). Modeling is essential for anisotropic properties as experimental measurements in many directions necessary to fully characterize anisotropy is not currently feasible for the majority of the temperature and pressure conditions found in the Earth's mantle.

In following, we will only discuss the elastic properties needed for seismic velocities, but the methods apply to all tensorial properties where the bulk property is governed by the volume fraction of the constituent minerals. Many properties of geophysical interest are of this type, for example thermal conductivity, thermal expansion, elasticity and seismic velocities. However these methods do not apply to properties determined by the connectivity of a phase, such as the electrical conductivity of rocks with conductive films on the grain boundaries (e.g. carbon). We will assume the sample may be microscopically heterogeneous due to grain size, shape, orientation or phase distribution, but will be considered macroscopically uniform. The complete structural details of the sample are in general never known, but a 'statistically uniform' sample contains many regions which are compositionally and structurally similar, each fairly representative of the entire sample. The local stress and strain fields at every point $\mathbf{r}$ in a linear elastic polycrystal which are completely determined by Hooke's law as follows

$$
\sigma_{\mathrm{ij}}(\mathbf{r})=\mathrm{C}_{\mathrm{ijkl}}(\mathbf{r}) \varepsilon_{\mathrm{kl}}(\mathbf{r})
$$

where $\sigma_{i j}(\mathbf{r})$ is the stress tensor, $C_{i j k l}(\mathbf{r})$ is the elastic stiffness tensor and $\varepsilon_{\mathrm{kl}}(\mathbf{r})$ the strain tensor at point $\mathbf{r}$. The evaluation of the effective constants of a polycrystal would be the summation of all components as a function of position, if we know the spatial functions of stress and strain. The average stress $\langle\sigma\rangle$ and strain $\langle\varepsilon\rangle$ of a statistically uniform sample are linked by an effective macroscopic modulus $C^{*}$ that obeys Hooke's law of linear elasticity,

$$
C^{*}=\langle\sigma\rangle\langle\varepsilon\rangle-1
$$

where

$$
\varepsilon=\frac{1}{V} \int \varepsilon(r) . d r
$$

and

$$
\sigma=\frac{1}{V} \int \sigma(r) \cdot d r
$$

and $\mathrm{V}$ is the volume, the notation <.> denotes an ensemble average. The stress $\sigma(\mathbf{r})$ and strain $\varepsilon(\mathbf{r})$ distribution in a real polycrystal varies discontinuously at the surface of grains. By replacing the real polycrystal with a 'statistically uniform' sample we are assuming that $\sigma(\mathbf{r})$ and strain $\varepsilon(\mathbf{r})$ are varying slowly and continuously with position $\mathbf{r}$.

A number of methods are available for determining the effective macroscopic modulus. We will briefly present these methods which try to take into account an increasing amount of microstructural information, which of course results in increasing theoretical complexity, but yields estimates which are closer to experimental values. The methods can be classified by using the concept of the order of the statistical probability functions used to quantitatively describe the microstructure (Kröner, 1978). A zero order bound is given when one has no statistical information on the microstructure of the polycrystal and for example we do not know the orientation of the component crystals, in this case we have to use the single crystal properties. The maximum and minimum of the single crystal property are the zero order bounds. The simplest and best known averaging techniques for obtaining estimates of the effective elastic constants of polycrystals are the Voigt (1928) and Reuss (1929) averages. These averages only use the volume fraction of each phase, the orientation and the elastic constants of the single crystals or grains. In terms of statistical probability functions, these are first order bounds as only the first order correlation function is used, which is the volume fraction. Note no information on the shape or position of neighboring grains is used. The Voigt average is found by simply assuming that the strain field is everywhere constant (i.e. $\varepsilon(\mathbf{r})$ is independent of $\mathbf{r}$ ). The strain at every position is set equal to the macroscopic strain of the sample. $\mathrm{C}^{*}$ is then 
estimated by a volume average of local stiffnesses $\mathrm{C}\left(\mathbf{g}_{\mathbf{i}}\right)$ with orientation $\mathbf{g}_{\mathbf{i}}$, and volume fraction $\mathrm{V}_{\mathrm{i}}$,

$$
C \approx C^{\text {voigt }}=\left[\sum_{i} \operatorname{Vi} . C(g i)\right]
$$

Reuss average is found by assuming that the stress field is everywhere constant. The stress at every position is set equal to the macroscopic stress of the sample. $\mathrm{C}^{*}$ or $\mathrm{S}^{*}$ is then estimated by the volume average of local compliances $\mathrm{S}\left(\mathbf{g}_{\mathbf{i}}\right)$,

$$
\begin{gathered}
C^{*} \approx C^{\text {Reuss }}=\left[\sum_{i} \operatorname{Vi.S}(g i)\right]^{-1} \\
S^{*} \approx S^{\text {Reuss }}=\left[\sum_{i} \operatorname{Vi.S}(g i)\right] \\
\mathrm{C}^{\text {Voigt }} \neq \mathrm{C}_{\text {Reuss }} \text { and C Voigt } \neq\left[\mathrm{S}_{\text {Reuss }}\right]^{-1}
\end{gathered}
$$

These two estimates are not equal for anisotropic solids with the Voigt being an upper bound and the Reuss a lower bound. A physical estimate of the moduli should lie between the Voigt and Reuss average bounds as the stress and strain distributions are expected to be somewhere between uniform strain (Voigt bound) and uniform stress (Reuss bound). Hill (1952) observed that arithmetic mean of the Voigt and Reuss bounds, sometimes called the Hill or Voigt-Reuss-Hill (VRH) average, is often close to experimental values. The VRH average has no theoretical justification. In Fig. 7a,b we present the Voigt, Reuss and Hill averages of a number of mantle minerals from Table 1 for randomly orientated polycrystals as a function of anisotropy for compressional $(\mathrm{Vp})$ and shear $(\mathrm{Vs})$ wave anisotropy. In Earth sciences, the Voigt, Reuss and Hill averages have been widely used for averages of oriented polyphase rocks (e.g. Crosson and Lin, 1971). Although the Voigt and Reuss bounds are often far apart for anisotropic materials, they still provide the limits within which the experimental data should be found.

Recently, several authors have searched for a geometric mean of oriented polycrystals using the exponent of the average of the natural logarithm of the eigenvalues of the stiffness matrix (Morawiec, 1989 ; Matthies and Humbert, 1993). Their choice of this averaging procedure was guided by the fact that the ensemble average elastic stiffness $\langle\mathrm{C}\rangle$ should equal the inverse of the ensemble average elastic compliances $\langle\mathrm{S}\rangle^{-1}$, which is not true, for example, of the Voigt and Reuss estimates. A method of determining the geometric mean for arbitrary orientation distributions has been developed (Matthies and Humbert, 1993). The method derives from the fact that a stable elastic solid must have an elastic strain energy which is positive. It follows from this
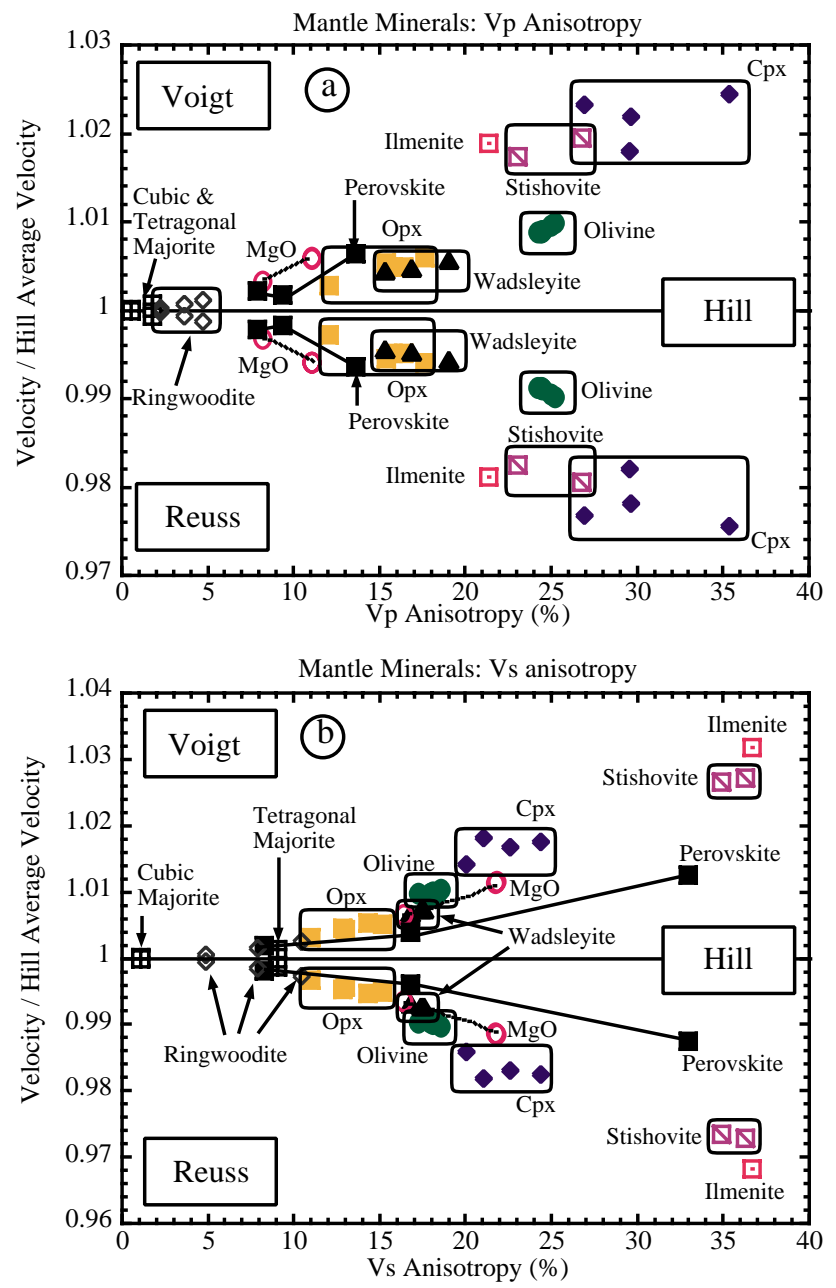

Figure 7. The P (a) and S wave (b) anisotropy for randomly oriented aggregates of mantle phases at room temperature and pressure given in Table 1. Note the large differences for the three values for perovskite, the most anisotropic value corresponds to the experimental 1994 determination and least anisotropic to the 1989. Note also the difference in Vp and Vs anisotropy for the minerals, for example Cpx has a particularly high Vp anisotropy and ilmenite a very high Vs.

that the eigenvalues of the elastic matrix must all be positive. Comparison between Voigt, Reuss, Hill and selfconsistent estimates show that the geometric mean provides estimates very close to the self-consistent method, but at considerably reduced computational complexity (Matthies and Humbert, 1993). The condition that the macroscopic polycrystal elastic stiffness $\langle\mathrm{C}\rangle$ must equal the inverse of the aggregate elastic compliance $\langle\mathrm{S}\rangle^{-1}$ would appear to be a powerful physical constraint on the averaging method (Matthies and Humbert, 1993). However, the arithmetic (Hill) and geometric means are very similar (Mainprice and 
Humbert, 1994), which tends to suggest that they are just mean estimates with no additional physical significance.

The second set of methods use additional information on the microstructure to take into account the mechanical interaction between the elastic elements of the microstructure. Mechanical interaction will be very important for rocks containing components of very different elastic moduli, such as solids, liquids, gases and voids. The most important approach in this area is the 'self-consistent' (SC) method (e.g. Hill, 1965). The SC method was introduced for materials with a high concentration of inclusions where the interaction between inclusions is significant. In the SC method, an initial estimate of the anisotropic homogeneous background medium of the polycrystal is calculated using the traditional volume averaging method (e.g. Voigt). All the elastic elements (e.g. grains, voids etc.) are inserted into the background medium using Eshelby's (1957) solution for a single ellipsoidal inclusion in an infinite matrix. The elastic moduli of the ensemble, inclusion and background medium are used as the 'new' background medium for the next inclusion. The procedure is repeated for all inclusions and repeated in an iterative manner for the polycrystal until a convergent solution is found. The interaction is notionally taken into account by the evolution of the background medium which contains information about the inclusions, albeit in an homogenous form. As the inclusion can have an ellipsoidal shape, an additional microstructural parameter is taken into account by this type of model.

Several people (e.g. Bruner, 1976; Henyey and Pomphrey, 1982) have remarked that the SC progressively overestimates the interaction with increasing concentration. They proposed an alternative differential effective medium (DEM) method in which the inclusion concentration is increased in small steps with a re-evaluation of the elastic constants of the aggregate at each increment. This scheme allows the potential energy of the medium to vary slowly with inclusion concentration (Bruner, 1976). Since the addition of inclusions to the background material is made in very small increments, one can consider the concentration step to be very dilute with respect to the current effective medium. It follows that the effective interaction between inclusions can be considered negligible and we can use the inclusion theory of Eshelby (1957) to take into account the interaction. In contrast, the SC uses Eshelby's theory plus an iterative evaluation of the background medium to take into account the interaction. Recently Mainprice (1997) has compared the results of SC and DEM for anisotropic oceanic crustal and mantle rocks containing melt inclusions and found the results to be very similar for melt fractions of less than $30 \%$. At higher melt fractions the SC exhibits a threshold value around $60 \%$ melt, whereas the DEM varies smoothly up a $100 \%$ melt. The presence of a threshold in the SC calculations is due to the specific way that the interaction is taken into account. The estimates of both methods are likely to give relatively poor results at high fractions of a phase with strong elastic contrast with the other constituents as other phenomena, such as mechanical localization related to the percolation threshold, are likely to occur.

The third set of methods uses higher order statistical correlation functions to take into account the first or higher order neighbor relations of the various microstructural elements. The factors that need to be statistically described are the elastic constants (determined by composition), orientation and relative position of an element. If the element is considered to be small relative to grain size, then grain shape and the heterogeneity can be accounted for the relative position correlation function. Nearest neighbors can be taken into account using 2-point correlation function, which is also called an auto-correlation function by some authors. If we use the "statistically uniform" sample introduced above, we are effectively assuming that all the correlation functions used to describe the microstructure up to order infinity are statistically isotropic, this is clearly a very strong assumption. In the special case where all the correlation functions up to order infinity are defined, Kröner (1977, 1978) has shown that the upper and lower bounds converge for the self-consistent method so that $\mathrm{C}^{\mathrm{SC}}=\left(\mathrm{S}^{\mathrm{Sc}}\right)^{-1}$. The statistical continuum approach (e.g. Beran, 1968) is the most complete description and has been extensively used for model calculations (e.g. Beran et al., 1996; Mason and Adams, 1999). Until recently it has been considered too complicated for practical application. With the advent of automated determination of crystal orientation and positional mapping using electron back-scattered diffraction (E.B.S.D.) in the scanning electron microscope (Adams et al., 1993), digital microstructural maps are now available for the determination of statistical correlation functions. This approach provides the best possible estimate of the elastic properties but at the expense of considerably increased computational complexity.

The fact that there is a wide separation in the Voigt and Reuss bounds for anisotropic materials is caused by the fact that the microstructure is not fully described by such methods. However, despite the fact that these methods do not take into account such basic information as the position or the shape of the grains, several studies have shown that the Voigt or the Hill average are within 5 to $10 \%$ of experimental values for low porosity rocks free of fluids. For example, Barruol and Kern (1996) showed for several anisotropic lower crust and upper mantle rocks from the Ivrea zone in Italy that the Voigt average is within 5\% of the experimentally measured velocity.

\section{THE CALCULATION OF ANISOTROPIC SEISMIC PROPERTIES}

Orientation of crystals in a polycrystal can be measured by volume diffraction techniques (e.g. X-ray or neutron diffraction) or individual orientation measurements (e.g. U- 
stage \& Optical microscope, electron channeling or EBSD). An orientation, often given the letter $\mathbf{g}$, of a grain or crystal in sample co-ordinates can be described by the rotation matrix between crystal and sample co-ordinates. In practice it is convenient to describe the rotation by a triplet of Euler angles, for example $\mathbf{g}=(\varphi 1 \phi \varphi 2)$ used by Bunge (1982). One should be aware there are many different definitions of Euler angles that are used in the physical sciences, here we will use the definition given by Bunge (1982). The orientation distribution function (O.D.F.) $\mathrm{f}(\mathrm{g})$ is defined as the volume fraction of orientations with an orientation in the interval between $\mathbf{g}$ and $\mathbf{g}+\mathrm{d} \mathbf{g}$ in a space containing all possible orientations given by

$$
\Delta \mathrm{V} / \mathrm{V}=\int \mathrm{f}(\mathbf{g}) \mathrm{d} \mathbf{g}
$$

where $\Delta \mathrm{V} / \mathrm{V}$ is the volume fraction of crystals with orientation $\mathbf{g}, \mathrm{f}(\mathrm{g})$ is the texture function and $\mathrm{d} \mathbf{g}=1 / 8 \pi^{2}$ $\sin \phi d \varphi 1 d \phi d \varphi 2$ is the volume of the region of integration in orientation space.

To calculate the seismic properties of a polycrystal, one must evaluate the elastic properties of the aggregate. In the case of an aggregate with a crystallographic fabric, the anisotropy of the elastic properties of the single crystal must be taken into account. For each orientation $\mathbf{g}$, the single crystal properties have to be rotated into the specimen coordinate frame using the orientation or rotation matrix $\mathrm{g}_{\mathrm{ij}}$,

$$
\mathrm{C}_{\mathrm{ijkl}}(\mathbf{g})=\mathrm{g}_{\mathrm{ip}} \cdot \mathrm{g}_{\mathrm{jq}} \cdot \mathrm{g}_{\mathrm{kr}} \cdot \mathrm{glt}_{\mathrm{gl}} \mathrm{C}_{\mathrm{pqrt}}\left(\mathrm{g}^{\mathrm{O}}\right)
$$

where $\mathrm{C}_{\mathrm{ijkl}}(\mathrm{g})$ is the elastic property in sample coordinates, $g_{i j}=g(\varphi 1 \phi \varphi 2)$ the measured orientation in sample co-ordinates and $\operatorname{Cpqrt}\left(\mathbf{g}^{\mathrm{O}}\right)$ is the elastic property in crystal co-ordinates.

The elastic properties of the polycrystal may be calculated by integration over all possible orientations of the ODF. Bunge (1985) has shown that integration is given as:

$$
<\mathrm{C}_{\mathrm{ijkl}}>^{\mathrm{m}}=\int \mathrm{C}_{\mathrm{ijkl}} \mathrm{m}^{\mathrm{m}}(\mathbf{g}) \cdot \mathrm{f}(\mathbf{g}) \mathrm{dg}
$$

where $\left\langle\mathrm{C}_{\mathrm{ijkl}}\right\rangle \mathrm{m}$ is the elastic properties of the aggregate of mineral $\mathrm{m}$. Alternatively it may be determined by simple summation of individual orientation measurements,

$$
<\mathrm{C}_{\mathrm{ijkl}}>\mathrm{m}=\Sigma \mathrm{C}_{\mathrm{ijkl}} \mathrm{m}^{\mathrm{m}}(\mathbf{g}) . \mathrm{v}(\mathbf{g})
$$

where $\mathrm{v}(\mathbf{g})$ is the volume fraction of the grain in orientation $\mathbf{g}$. For example, the Voigt average of the rock for $\mathrm{m}$ mineral phases of volume fraction $\mathrm{v}(\mathrm{m})$ is given as

$$
\left\langle\mathrm{C}_{\mathrm{ijkl}}>\mathrm{Voigt}=\Sigma \mathrm{v}(\mathrm{m})<\mathrm{C}_{\mathrm{ijkl}}\right\rangle^{\mathrm{m}}
$$

The final step is the calculation of the three seismic phase velocities by the solution of the Christoffel equation,

$$
\mid<C_{i j k l}>\text { Voigt } n_{j} n_{l}-\rho V^{2} \delta_{i k} \mid=0
$$

where $\mathrm{n}$ is the plane wave propagation direction, $\rho$ is the density of the rock, $\delta_{\mathrm{ik}}$ is the Kronecker delta and the three values of $\mathrm{V}$ are the three seismic phase velocities.

To calculate the elastic constants at pressures and temperatures of Earth's mantle the single crystal elastic constants are extrapolated to pressure and temperature using the following relationship:

$$
\begin{gathered}
\mathrm{C}_{\mathrm{ij}}(\mathrm{PT})=\mathrm{C}_{\mathrm{ij}}\left(\mathrm{P}_{\mathrm{O}} \mathrm{T}_{\mathrm{O}}\right)+\left(\mathrm{dC} \mathrm{C}_{\mathrm{ij}} / \mathrm{dP}\right) \cdot\left(\mathrm{P}-\mathrm{P}_{\mathrm{O}}\right)+1 / 2 \\
\left(\mathrm{~d}^{2} \mathrm{C}_{\mathrm{ij}} / \mathrm{dP}^{2}\right) \cdot\left(\mathrm{P}-\mathrm{P}_{\mathrm{O}}\right)^{2}+\left(\mathrm{dC} \mathrm{C}_{\mathrm{ij}} / \mathrm{dT}\right) \cdot\left(\mathrm{T}-\mathrm{T}_{\mathrm{O}}\right)
\end{gathered}
$$

where $\mathrm{Cij}(\mathrm{PT})$ are the elastic constants at Earth's mantle pressure $\mathrm{P}$ and temperature $\mathrm{T}, \operatorname{Cij}\left(\mathrm{P}_{\mathrm{O}} \mathrm{T}_{\mathrm{O}}\right)$ the elastic constants at reference pressure $\mathrm{P}_{\mathrm{O}}=0.1 \mathrm{MPa}$ and temperature $\mathrm{T}_{\mathrm{O}}=25{ }^{\circ} \mathrm{C} ; \mathrm{dCij} / \mathrm{dP}$ is the first order pressure derivative and $\mathrm{dCij} / \mathrm{dT}$ is the first order temperature derivative. The second order pressure derivatives $\mathrm{d}^{2} \mathrm{Cij} / \mathrm{dP}^{2}$ are available for an increasing number of mantle minerals (e.g. olivine, orthopyroxene, garnet, $\mathrm{MgO}$ ) and first order temperature derivatives seem to adequately describe the temperature dependence of most minerals. Note however when phase transitions occur, the specific changes in elastic constants at pressures near the phase transition will have to be taken into account, for example the $\mathrm{SiO}_{2}$ polymorphs. The seismic velocities also depend on the density of the minerals at pressure and temperature which can be calculated using an appropriate equation of state (Knittle, 1995). The Murnaghan equation of state derived from finite strain is sufficiently accurate at moderate compression (Knittle, 1995) of the upper mantle and leads to the following expression for density as a function of pressure,

$$
\rho(P)=\rho_{O}\left(1+\left(K^{\prime} / K\right) \cdot\left(P-P_{O}\right)\right)^{1 / K^{\prime}}
$$

where $\mathrm{K}$ is bulk modulus, $\mathrm{K}^{\prime}=\mathrm{dK} / \mathrm{dP}$ the pressure derivative of $K, \rho_{O}$ is the density at reference pressure $P_{O}$ and temperature $\mathrm{T}_{\mathbf{O}}$. For temperature the density varies as

$$
\rho(T)=\rho_{\mathrm{o}}\left[1-\int \alpha_{\mathrm{V}}(\mathrm{T}) \mathrm{dT}\right] \approx \rho_{\mathrm{o}}\left[1-\alpha_{\mathrm{aV}}(\mathrm{T}-\mathrm{To})\right]
$$

where $\alpha_{\mathrm{V}}(\mathrm{T})=1 / \mathrm{V}(\partial \mathrm{V} / \partial \mathrm{T})$ is the volume thermal expansion coefficient as a function of temperature and $\alpha_{\mathrm{av}}$ is 
an average value of thermal expansion which is constant over the temperature range (Fei, 1995). According to Watt (1988) an error of less $0.4 \%$ on the $\mathrm{P}$ and $\mathrm{S}$ velocity results from using $\alpha_{\mathrm{av}}$ to $1100 \mathrm{~K}$ for $\mathrm{MgO}$. For temperatures and pressures of the mantle, the density is described for this paper by,

$$
\rho(\mathrm{P}, \mathrm{T})=\rho_{\mathrm{O}}\left\{\left(1+\left(\mathrm{K}^{\prime} / \mathrm{K}\right) \cdot\left(\mathrm{P}-\mathrm{P}_{\mathrm{O}}\right)\right)^{1 / \mathrm{K}^{\prime}}\left[1-\alpha_{\mathrm{av}}(\mathrm{T}-\mathrm{To})\right]\right\}(16)
$$

\section{CRYSTAL PREFERRED ORIENTATION AND SEISMIC ANISOTROPY OF THE MANTLE}

The LPO in rocks of upper mantle origin is now well established (e.g. Mercier, 1985; Nicolas and Christensen, 1987). Recently Ben Ismail and Mainprice (1998) created a database of olivine LPO patterns from a variety of the upper mantle geodynamic environments (ophiolites, subduction zones, and kimberlites) with a range of micro-structures. The database shows a large variation of fabric patterns. Four types of fabric pattern symmetry have been distinguished. Sample 900A65A (Fig. 8) is characterized by a point maxima distribution of [100], [010] and [001] parallel to the $\mathrm{X}$ (lineation), Z (pole to foliation) and Y structural directions respectively. Sample DC45 is the partial "fiber texture" (Bunge, 1982) which has axial symmetry with the [100] as a rotational axis of symmetry with [010] and [001] forming girdles in the (YZ) structural plane normal to the lineation. This type may have maxima of [010] and [001] parallel respectively either to the $\mathrm{Y}$ and $\mathrm{Z}$ directions as in DC45, or to the $\mathrm{Z}$ and $\mathrm{Y}$ directions. FRB1309 type of fiber texture has [010] as symmetry axis parallel to the foliation pole (Z) with [100] and [001] form girdles in the XY foliation plane. JJG2513 type of fiber texture has [001] as symmetry axis parallel to Y direction with [100] and [010] forming girdles in the XZ plane. Of these LPOs 900A65A, DC45 and FRB1309 are the most common whereas JJG2513 is quite rare. Another very rare pattern is shown by PHN1611 (Mercier and Carter, 1975) which has a point maximum of [010] parallel to $\mathrm{Y}$ and [100] and [001] at $45^{\circ}$ to $\mathrm{X}$ and $\mathrm{Z}$ in the XZ plane. This sample comes from kimberlite mylonites formed during the extraction process and is not thought to be representative of the upper mantle.

All LPOs in Fig. 8 result in high Vp velocities near the lineation (X) (Fig. 9). For the more common fabrics the maximum shear wave splitting anisotropy is somewhere in the $\mathrm{YZ}$ plane normal to the lineation (X), often close to $\mathrm{Y}$ (e.g. DC45 and FRB109) (Fig. 9). The shear wave anisotropy of the JJG2513 LPO is more complex with a maximum near the foliation normal (Z). However, all olivine LPO result in the fastest (Vs1) $S$ wave being polarized in zone axis parallel to the high concentration of [100] axes (e.g. 900A65A). In general, the Vs1 polarization is parallel, or nearly parallel, to the foliation (XY) plane as the high concentration of [100] axes is near the lineation (X).
Enstatite (orthopyroxene) fabrics are characterized by having a high concentration of [001] axes near the lineation (X) (Fig. 10). The variation of enstatite is similar to olivine with a single crystal type pattern (FB805), girdles of [010] and [001] in the (XY) foliation with [100] point maximum (KBBF4) or girdles of [100] and [010] in the YZ plane with [001] point maximum parallel to the lineation (X) (M57). The FB805 fabric is the most common opx fabric type. These opx fabrics result in a Vp maximum orientation which is much more variable than olivine. The $\mathrm{Vp}$ maximum is somewhere in the $\mathrm{XZ}$ plane, being more often close to the foliation normal (FB805, KBBF4), but can be near the lineation (X) (M57). However, the $\mathrm{Vp}$ minimum is always close to the $\mathrm{Y}$ direction (Fig. 11). The $\mathrm{S}$ wave maximum anisotropy is also very variable in orientation being somewhere in the $\mathrm{YZ}$ plane, normal to the lineation $(\mathrm{X})$. The $\mathrm{S}$-wave minimum anisotropy is always close to the lineation (X). All opx LPO result in the fastest $S$ wave (Vs1) being polarized in zone axis parallel to the high concentration of [001] axes (e.g. M57). In general, the Vs1 polarization is parallel, or nearly parallel, to the foliation (XY) plane as the high concentration of [001] axes is near the lineation $(\mathrm{X})$.

When olivine and opx are combined in a rock with these LPOs the effects are different for Vp and Vs anisotropy. The maximum $\mathrm{Vp}$ velocities of olivine and opx are typically at right angles (e.g. 900A65A, DC45) which causes a lowering of the overall $\mathrm{Vp}$ anisotropy and a change from orthorhombic to hexagonal symmetry when summed. On the other hand, the minimum $\mathrm{dVs}$ for both minerals is close to the lineation. The maximum $\mathrm{dVs}$ is in the $\mathrm{YZ}$ plane for both minerals. The Vs1 polarization is approximately parallel to the foliation in both minerals. When olivine and opx are combined, their S-wave characteristics are constructively combined.

Our statistical knowledge of mantle cpx fabrics is rather poor. We illustrate a pattern of diopside in Fig. 10 with [100] close to $\mathrm{Y}$ [010] close to the foliation normal (Z) and [001] close the lineation (X) (Barruol and Mainprice, 1993). The pattern is similar to many omphacites (sodic pyroxene) from eclogites (e.g. Godard and van Roermund, 1995). The cpx fabric results in $\mathrm{Vp}$ maximum at $\mathrm{Y}$ whereas olivine and opx tend to have a $\mathrm{Vp}$ minimum in this direction. The dVs maximum for cpx is parallel to the lineation $(\mathrm{X})$ whereas both have $\mathrm{dVs}$ minimum in this direction. The Vs1 polarization is a more complex pattern than either olivine or opx, being oblique to the foliation by $30^{\circ}$ or more. Cpx interferes destructively with both $\mathrm{Vp}$ and $\mathrm{Vs}$ of olivine and opx. Olivine and opx are in destructive interference for $\mathrm{Vp}$, but are constructive for Vs. Hence Vp anisotropy will vary a lot as a function of composition in Olivine, Opx and Cpx. Vs anisotropy will be fairly constant for differing proportions of olivine and opx, however changing the fraction of Cpx will vary the Vs anisotropy.

Several factors will affect the magnitude of anisotropy. The factors we have considered so far are mineralogy, 


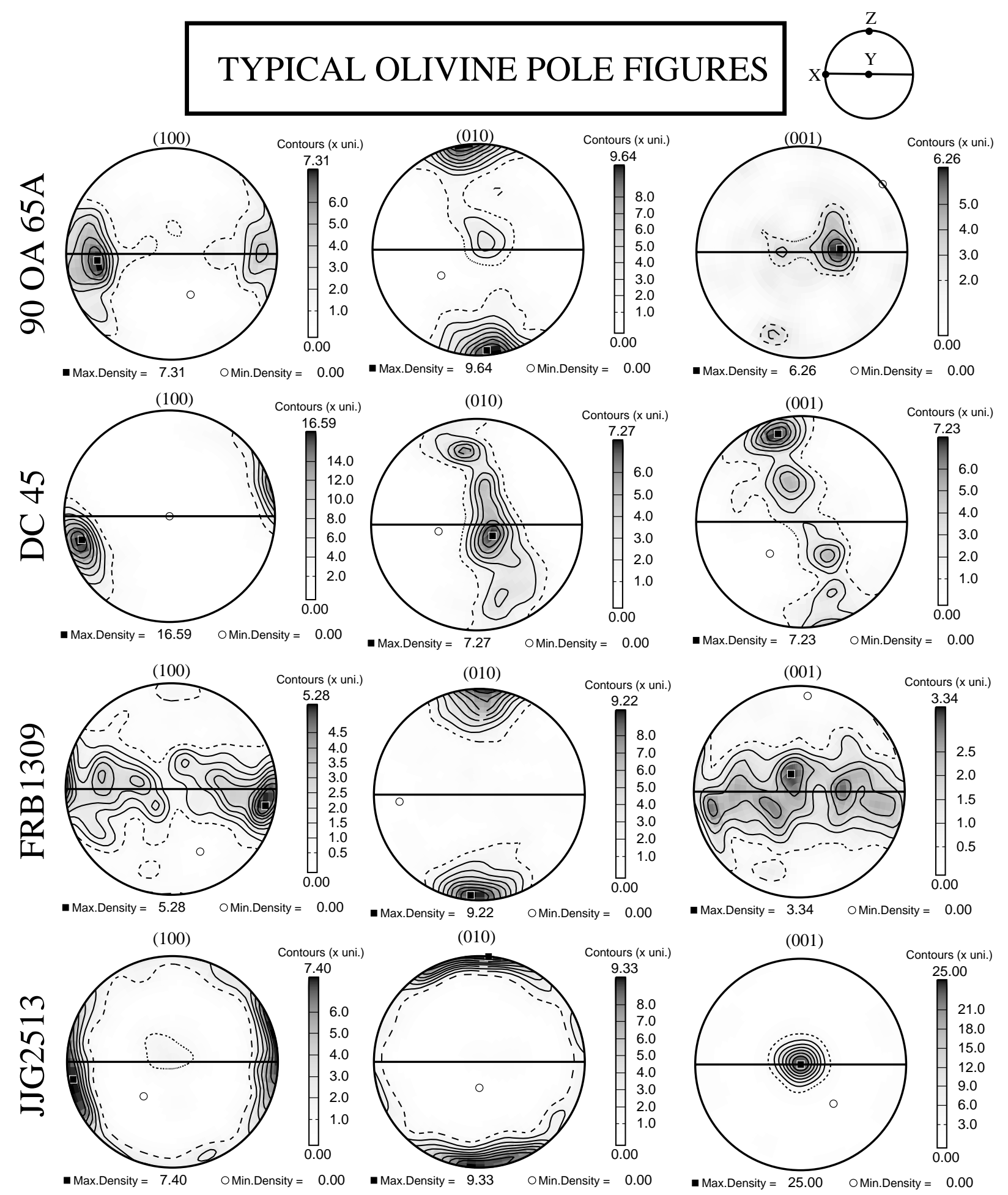

Figure 8. Olivine pole figures for [100], [010] and [001] from four typical samples. Contours in multiples of a uniform distribution. $\mathrm{X}=$ lineation, $\mathrm{Z}$ = pole to the foliation. Horizontal line is trace of the foliation plane. 


\section{OLIVINE SEISMIC ANISOTROPY}
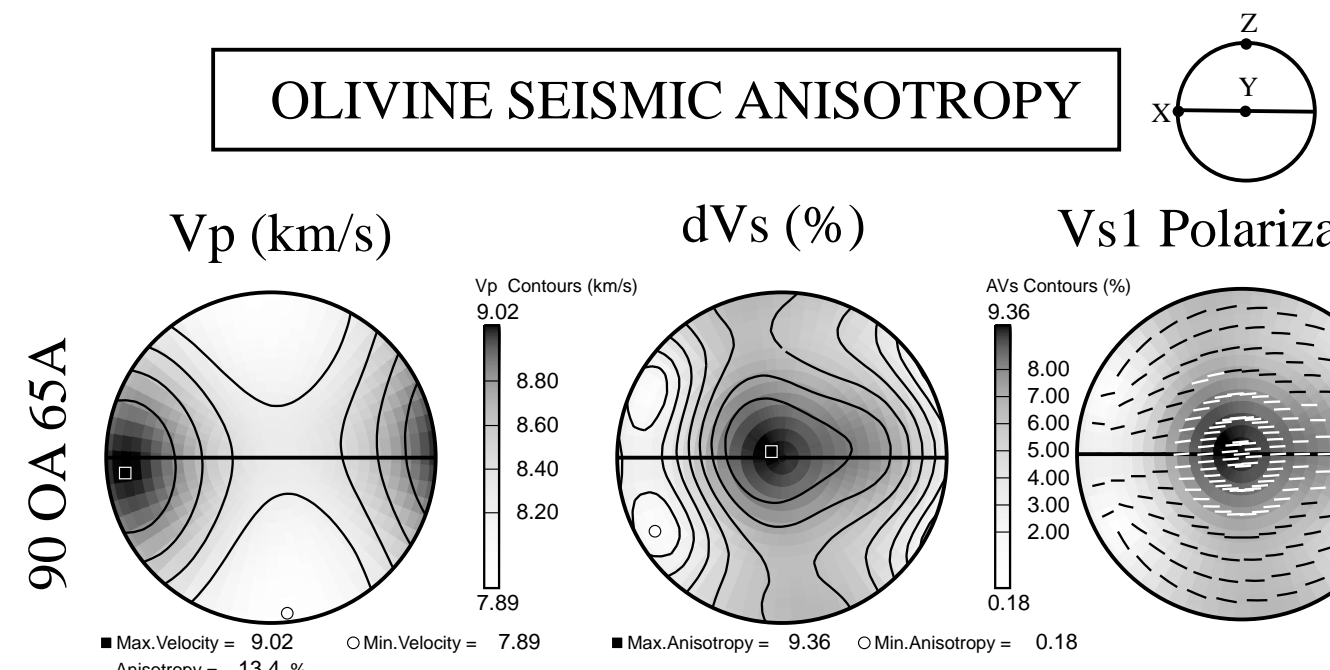

Vs1 Polarization
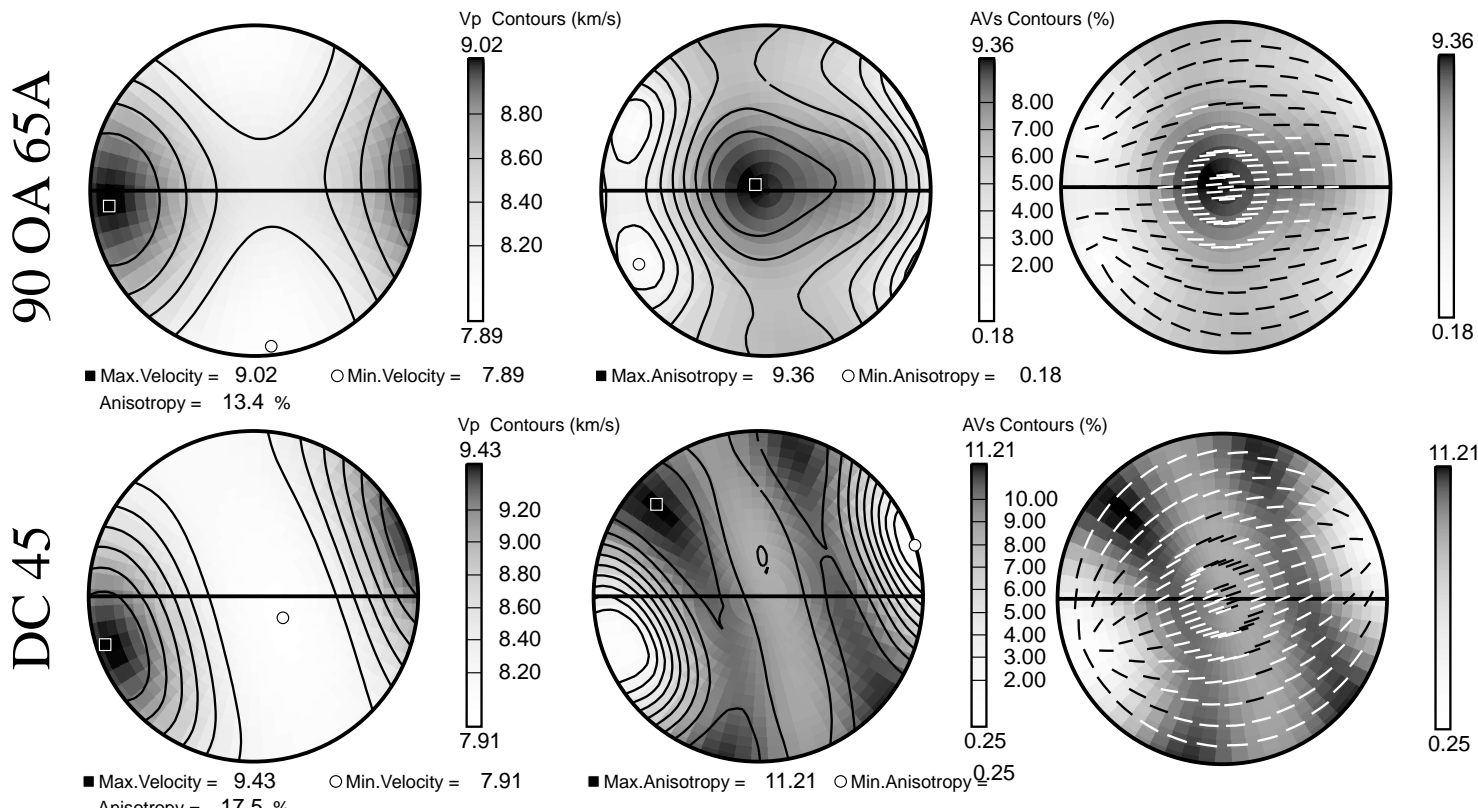

Contours $(\mathrm{km} / \mathrm{s})$

Max.Anisotropy $=9.36 \quad$ OMin.Anisotropy $=0.18$

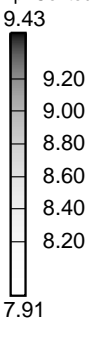
Anisotropy $=17.5 \%$
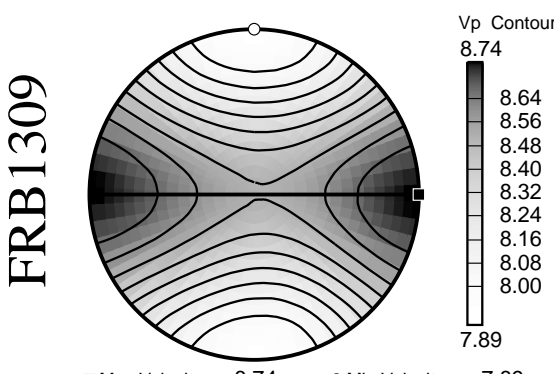
Anisotropy $=10.2 \%$
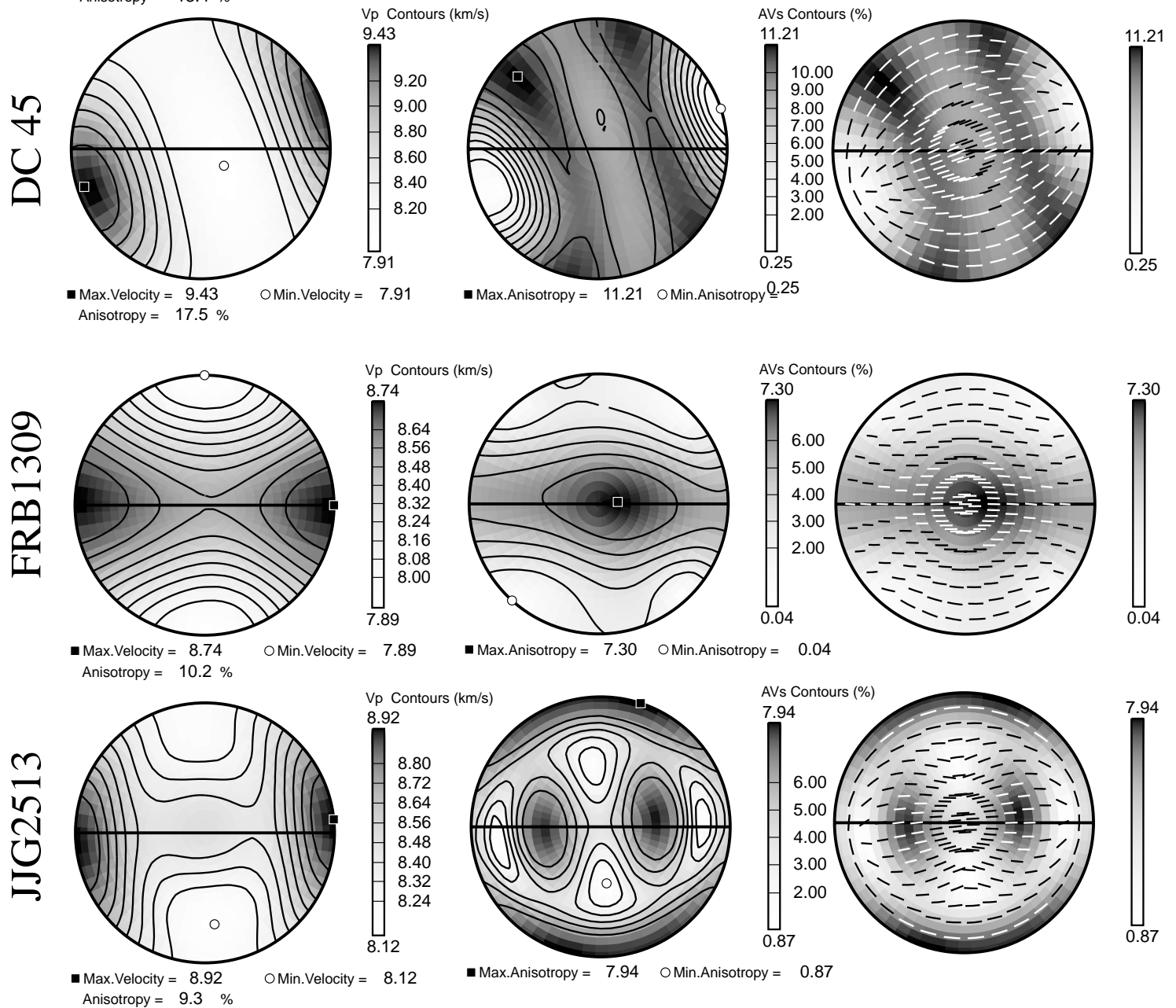

- Max.Anisotropy = 11.21 OMin.Anisotropy 0.25
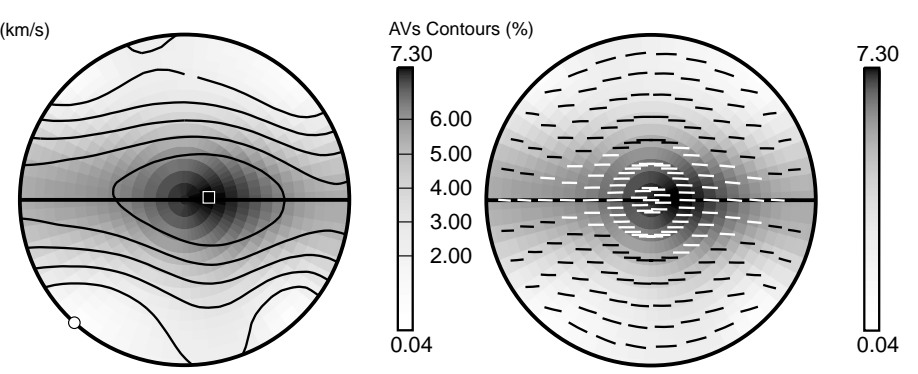

- Max.Anisotropy $=7.30 \quad$ OMin.Anisotropy $=0.04$
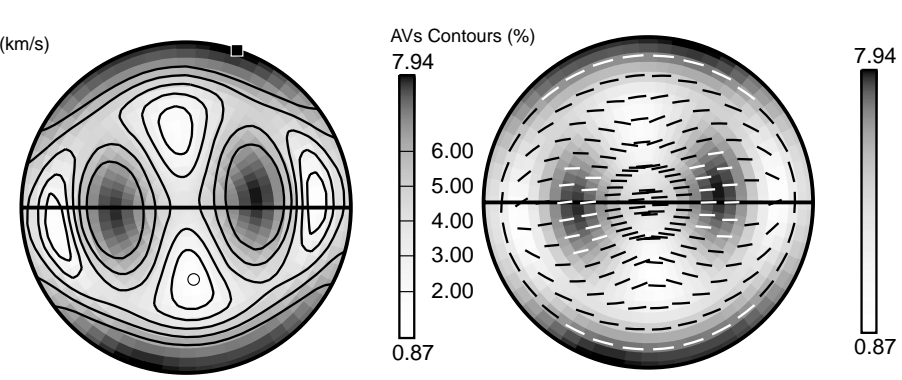

- Max.Anisotropy $=7.94 \quad$ OMin.Anisotropy $=0.87$

Figure 9. Olivine seismic anisotropy corresponding to the LPO given in Figure 8. Contours for Vp in $\mathrm{km} / \mathrm{s}$, dVs in $\%$ anisotropy and trace of the Vs1 polarization plane.

temperature and pressure dependence of the elastic constants, LPO and of course the orientation of the crystal or aggregate with respect to the propagation direction. It is well established that strength of LPO is a function of finite strain and hence the seismic anisotropy should evolve with deformation history. To quantify the degree of LPO Mainprice and Silver (1993) used the J index, which is defined as:

$$
J=\int f(g)^{2} d g
$$




\section{TYPICAL ORTHOPYROXENE AND CLINOPYROXENE POLE FIGURES}
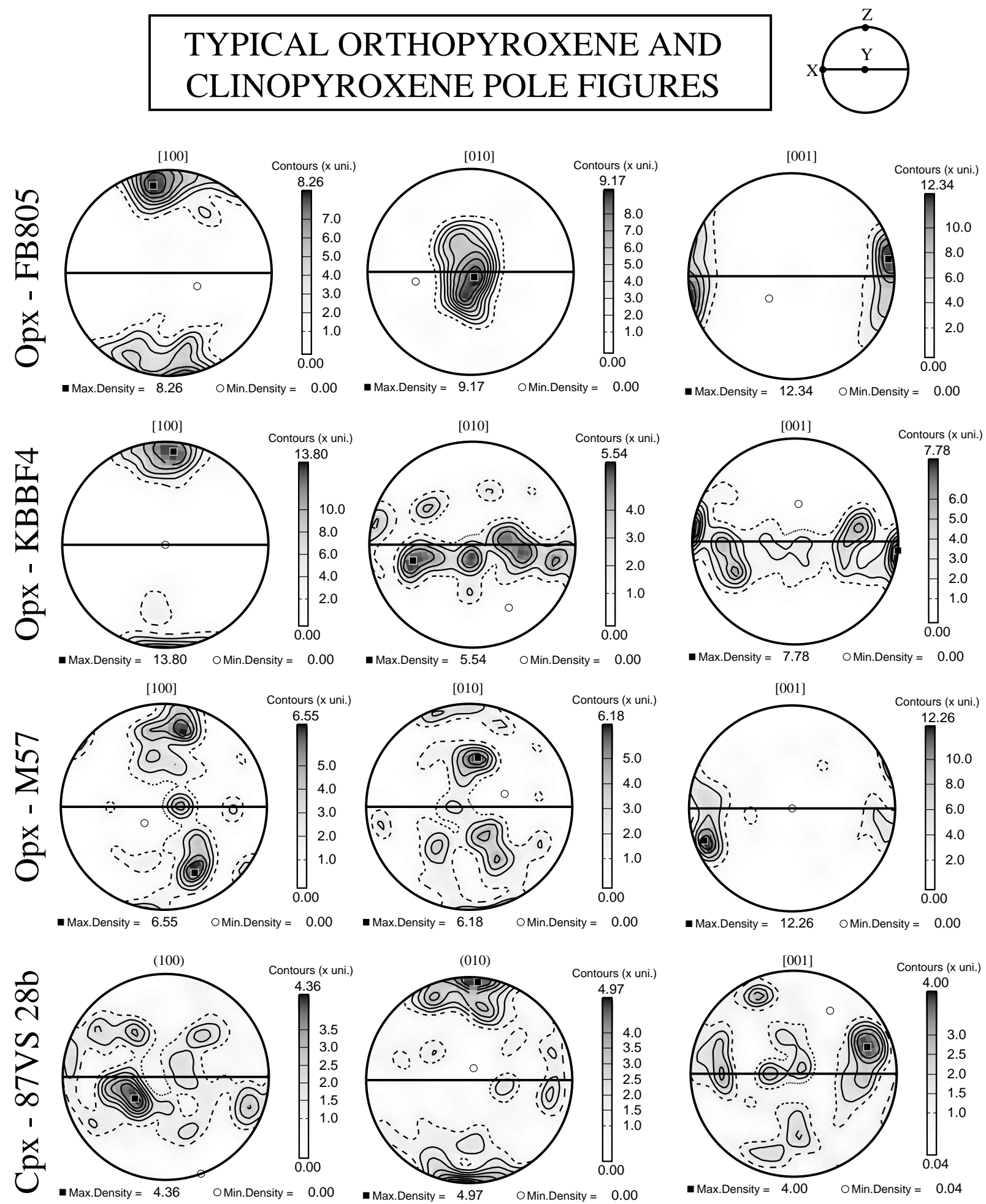

Figure 10. Orthopyroxene (enstatite) pole figures for [100], [010] and [001]. Clinopyroxene (diopside) pole figures for (100), (010) and [001]. Contours in multiples of a uniform distribution.

The $\mathrm{J}$ index has a value of one for random distribution of crystal orientations. Mainprice and Silver (1993) showed that $\mathrm{J}$ index and seismic anisotropy increased with increasing axial shorting of olivine aggregates by using the experimental data of Nicolas et al. (1973). They also undertook some numerical simulations in axial compression and simple shear using the kinematic model of Etchecopar and Vasseur (1987). A more extensive study of the methods used for numerical models of LPO (Tommasi et al., 1999 submitted) confirms that the $\mathrm{J}$ index increases with finite strain (Fig. 12). This study also shows that the numerical calculations can reproduce the LPO evolution of 


\title{
ORTHOPYROXENE \& CLINOPYROXENE SEISMIC ANISOTROPY
}
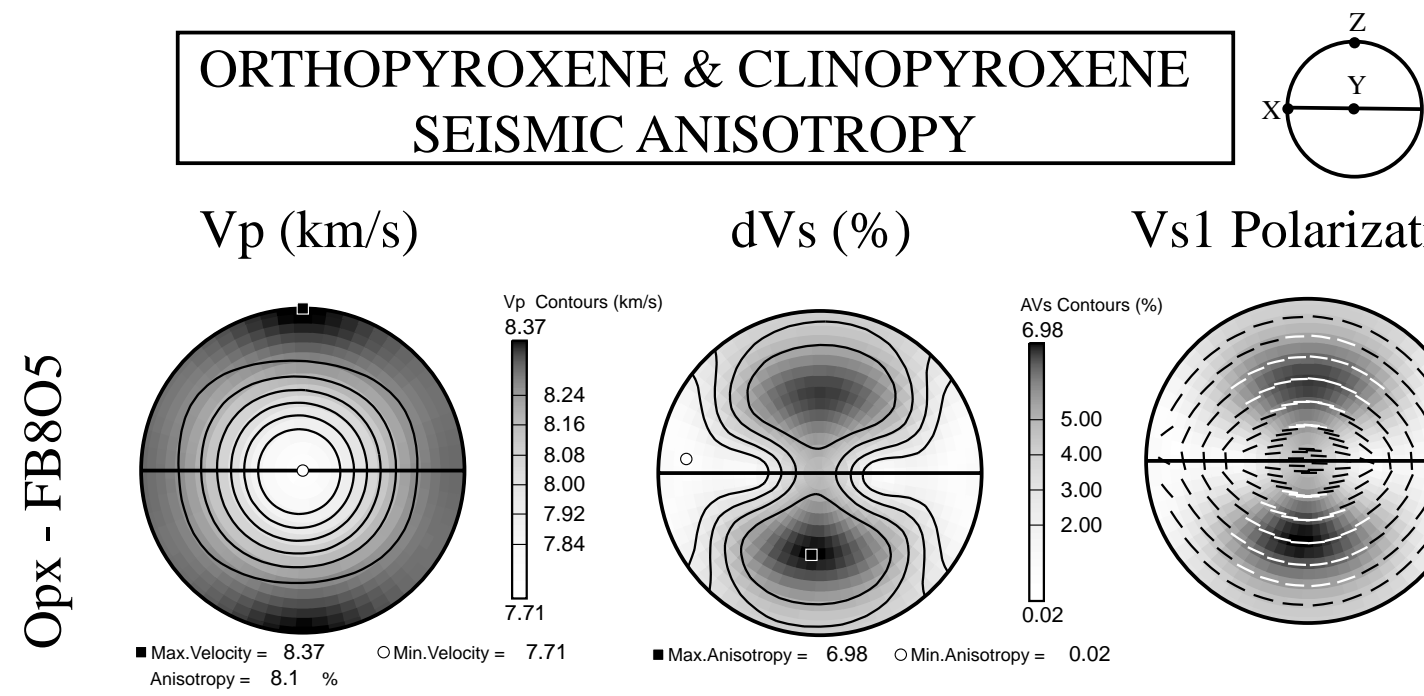

\author{
$\mathrm{dVs}(\%)$
}

Vs1 Polarization
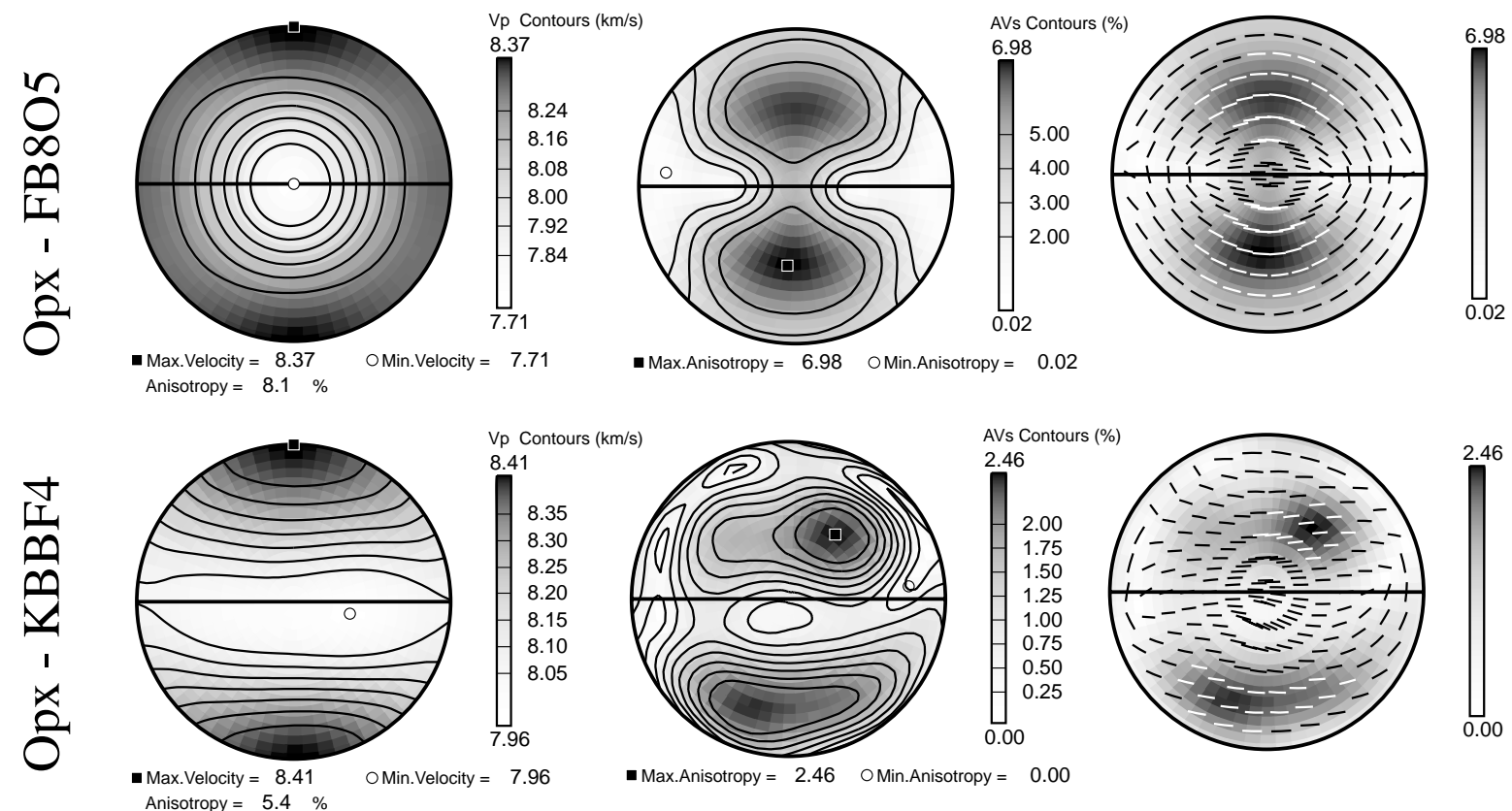

- Max.Anisotropy $=6.98 \quad$ OMin.Anisotropy $=0.02$ Anisotropy $=5.4 \%$
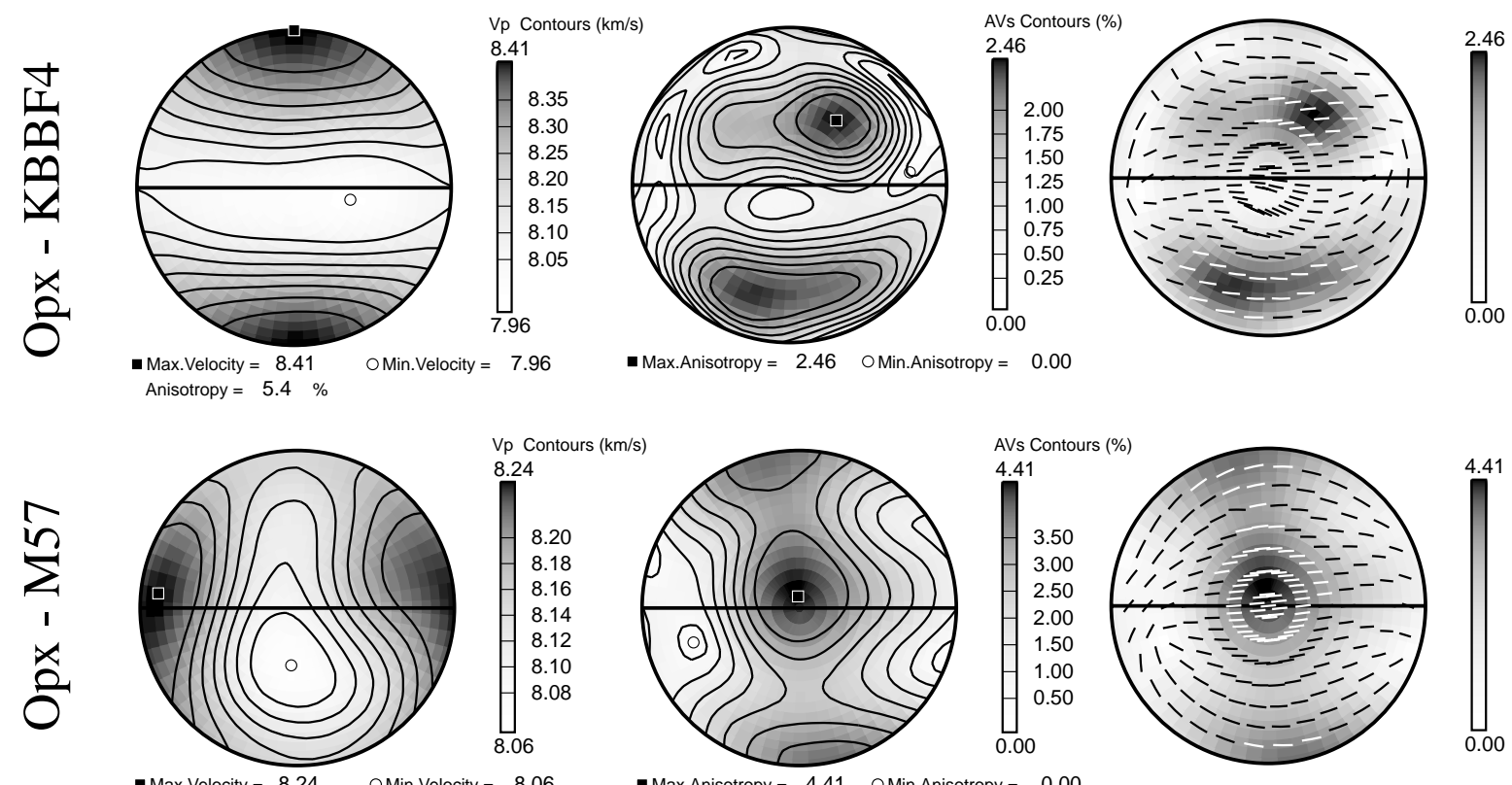

- Max.Anisotropy $=2.46 \quad$ OMin.Anisotropy $=0.00$
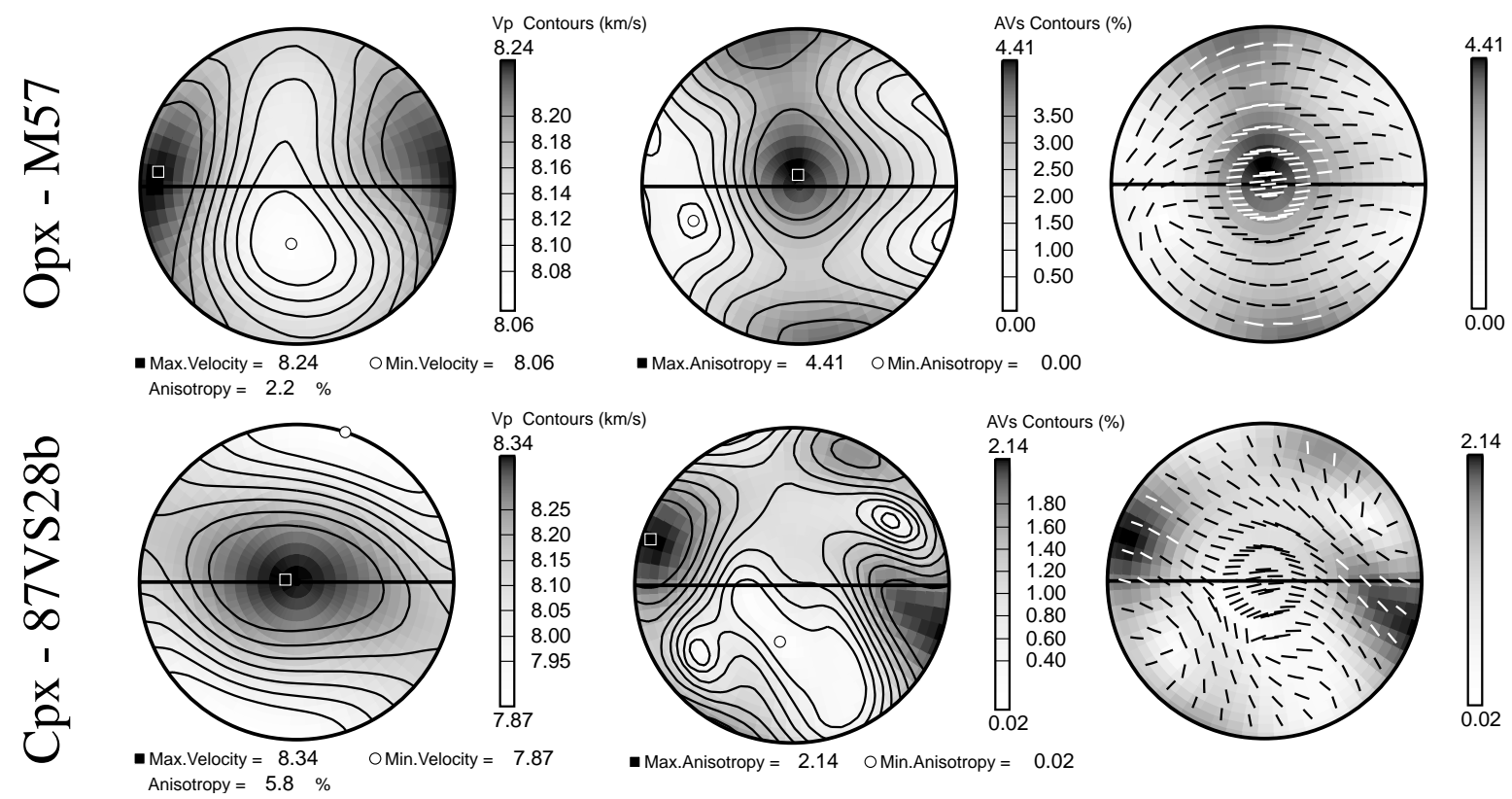

- Max.Anisotropy $=4.41 \quad$ OMin.Anisotropy $=0.00$
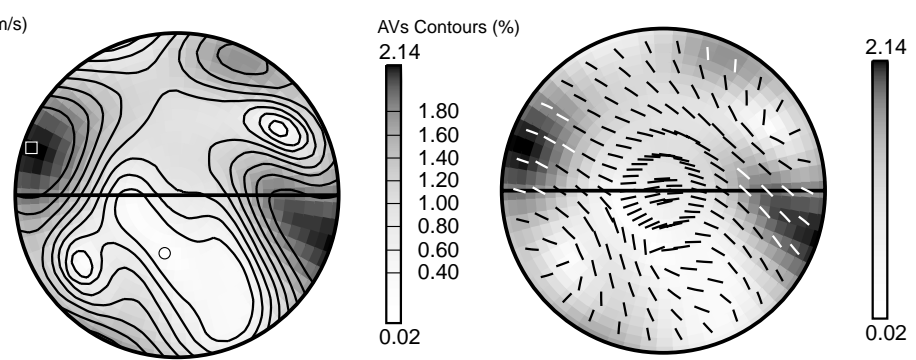

Figure 11. Orthopyroxene (enstatite) and clinopyroxene (diopside) seismic anisotropy corresponding to the LPO given in Figure 10. Contours for $\mathrm{Vp}$ in $\mathrm{km} / \mathrm{s}$, dVs in \% anisotropy and trace of the Vs1 polarization plane.

experimentally deformed olivine in simple shear (Zhang and Karato, 1995) and axial compression (Nicolas et al., 1973). Although the effects of recrystallization on LPO warrants further study, the patterns produced by the simulations are sufficiently good to capture the essentials of the seismic anisotropy for geodymanic modeling. For example, Chastel et al. (1993), Blackman et al. (1996), Blackman and Kendall (1997) have used the stress equilibrium (lower bound) 
method for modeling large scale mantle flow. Although this method produces olivine pole figures which are a less good match to the experimental or natural ones than the more computationally demanding self-consistent method (Wenk et al., 1991, Tommasi et al., 1999), there is little difference between the simulated seismic properties by these methods. Seismic anisotropy is sensitive to distribution of crystal orientations, but not to the details of individual pole figures because the seismic properties vary smoothly whereas the distribution of crystal axes can vary quite sharply with direction.

Ben Ismaïl and Mainprice (submitted, 1999) have recently established an orthopyroxene LPO database. Although for olivine it is straightforward to measure 100 or more crystals in a thin section to obtain a reliable measure of LPO, the low volume fraction (typically 30\%) of orthopyroxene makes this often impossible. Ben Ismail and Mainprice have developed a method to weight the ODF so that it is independent of the number of grains measured. Using this method, the orientations from the olivine and orthopyroxene databases were used to illustrate the variation of seismic anisotropy with LPO strength (Fig. 13). The relation between LPO strength and seismic anisotropy is non-linear for both olivine and opx with a steep increase in anisotropy at low $\mathbf{J}$ index followed by a slow increase from $\mathbf{J}$ index of about 12. The most remarkable thing is that olivine

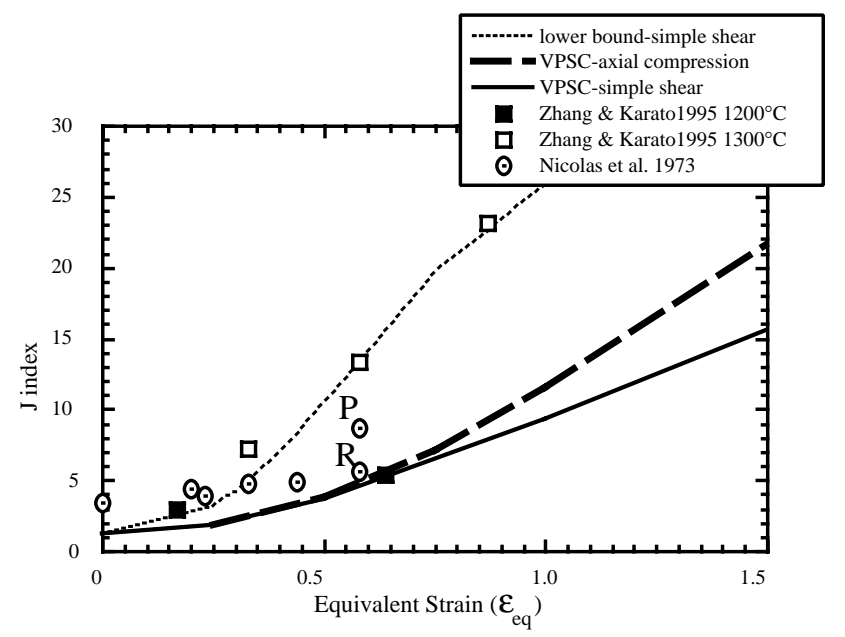

Figure 12. The LPO intensity (defined by the $\mathrm{J}$ index) as a function of equivalent strain, $\varepsilon_{\mathrm{eq}}=\sqrt{ }\left(2 / 3 \varepsilon_{\mathrm{ij}} \cdot \boldsymbol{\varepsilon}_{\mathrm{ij}}\right)$ where $\boldsymbol{\varepsilon}_{\mathrm{ij}}$ is the finite strain tensor. Axial strain $\varepsilon_{11}=\varepsilon_{\mathrm{eq}}$ and shear strain $\gamma=\sqrt{ } 3 \varepsilon_{\text {eq. }}$. Experiments of Nicolas et al. (1973) in axial compression (open circles, $\mathrm{P}$ porphyroclasts, $\mathrm{R}$ recrystallized grains). Experiments of Zhang and Karato (1985) in simple shear $\left(1200^{\circ} \mathrm{C}\right.$ filled squares, $1300^{\circ} \mathrm{C}$ open squares). Numerical simulations of Tommasi et al. (1999) (Equilibrium based "lower bound" - simple shear dashed line, VPSC - simple shear black line, VPSC - axial compression gray line).

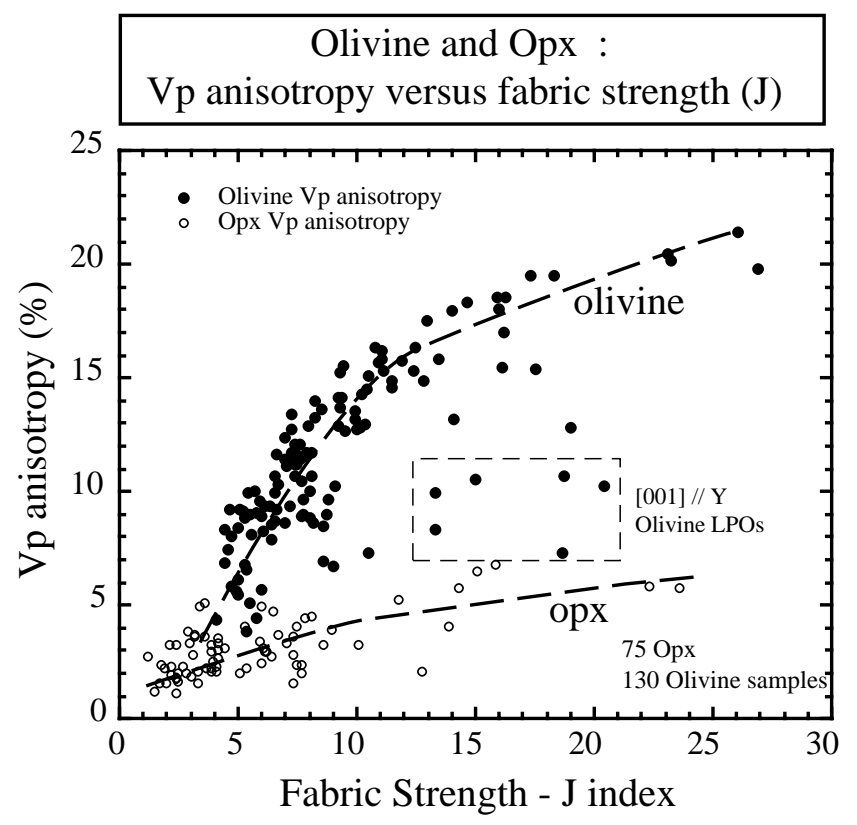

Figure 13. The Vp anisotropy (\%) as a function of LPO intensity (defined by the $\mathrm{J}$ index) for the olivine and opx databases. The olivine values in the black box are fabrics with a strong c-axes maximum parallel to $\mathrm{Y}$ such as JJG2513 in figure 8 . Trend lines are there to indicate tendencies and are not fitted functions.

is two to three times more anisotropic than opx for a given value of LPO strength. Olivine can develop very strong LPOs which have near single crystal anisotropy. Opx on the other hand is never more than half the single crystal anisotropy and in general is characterized by weaker LPOs ( $\mathrm{J}$ $=1$ to 10). In the database, the weakest olivine LPO has about $\mathbf{J}$ of 4 . There is a group of olivine fabrics from South African kimberlites with fabrics like JJG2513 which have an almost constant seismic anisotropy (ca. $9 \%$ for $\mathrm{Vp}$ ) despite a $\mathrm{J}$ index that varies from 12 to 20 . In naturally deformed peridotites there are no reliable markers of finite strain. If we take the $\mathbf{J}$ values of the olivine samples (about 5-25) and compare that with simulation or experiments in simple shear (Fig. 12), then it would appear that the LPO of naturally deformed peridotites is recording a shear strain gamma of between 0.8 and 2.5 with the majority being about 1.7 . One would expect that these rocks have suffered much high shear strains than 2.5 and the most plausible reason for the low values is that dynamic recrystallization reduces LPO intensity to maintain constant $\mathbf{J}$ values during deformation. Indeed, the recrystallized grains in the experiments of Nicolas et al. (1973) have weaker LPO than porphyroclasts (Fig. 12). However, effects such as grain boundary migration may increase the LPO strength. For example the strongest fabrics in the olivine database come from dunite samples with 


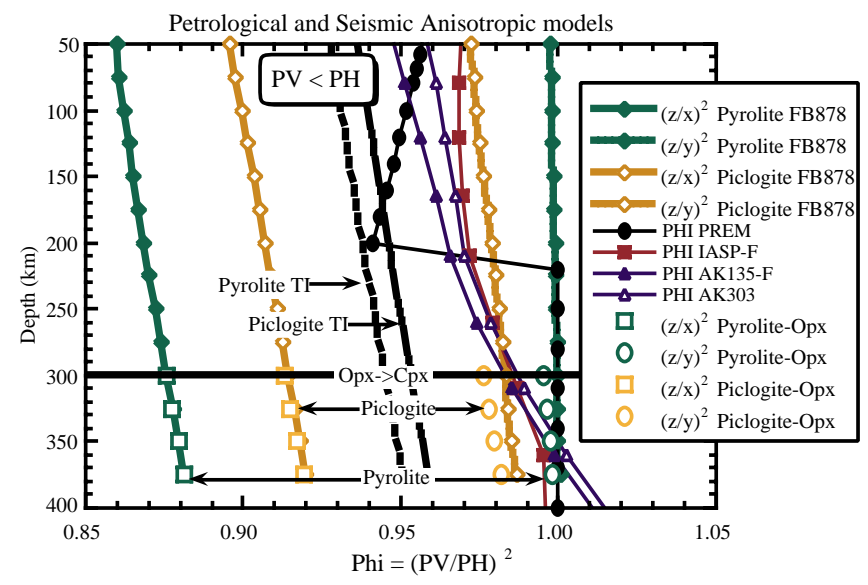

Figure 14. The $\mathrm{P}$ wave anisotropy parameter $\Phi=\left(\mathrm{V}_{\mathrm{PV}} / \mathrm{V}_{\mathrm{PH}}\right)^{2}$ as a function of depth for pyrolite and piclogite petrological compositions (see fig.5). The foliation plane (XY) is horizontal and the LPO of olivine and opx given by sample FB878 (not illustrated) and the cpx LPO by 87VS28b (Fig.10) and are constant with depth. The arithmetic mean of the upper $\left(\mathrm{V}_{\mathrm{Pz}} / \mathrm{V}_{\mathrm{Px}}\right)^{2}$ and the lower $\left(\mathrm{V}_{\mathrm{Pz}} / \mathrm{V}_{\mathrm{Py}}\right)^{2}$ bound is taken the transverse isotropic (TI) model marked by the black dashed lines. The modified model below $300 \mathrm{~km}$ opx $\rightarrow$ cpx transition is shown as open symbols. The global seismic models (Montagner and Kennett, 1996) are given for reference.

strong grain growth microstructures and the strongest fabrics from Zhang and Karato (1995) simple shear experiments on pure olivine aggregates at $1300^{\circ} \mathrm{C}$ also show evidence of grain growth. In most natural samples, the presence of other minerals (opx, garnet, cpx etc.) will inhibit extensive grain boundary migration. Hence it would seem likely that dynamic recrystallization by a mechanism such as sub-grain rotation would cause the LPO intensity and hence the seismic anisotropy to stabilize. Numerical simulation models that take into account recrystallization need to be applied to olivine (e.g. Wenk et al., 1997). If we take the value of anisotropy at the change in slope of anisotropy versus $\mathbf{J}$ index to represent the stabilized value, then database indicates that this occurs at about $60 \%$ of the anisotropy of the single crystal value for olivine and $35 \%$ for opx. Olivine will always display a stronger anisotropy than opx for two reasons; a) olivine has a stronger intrinsic single crystal anisotropy and b) the organization of the LPO patterns produce anisotropies that are typically $60 \%$ of the single crystal value whereas opx will only generate $35 \%$.

To estimate the variation of anisotropy in the upper mantle as a function of depth, we have taken a sample (FB878) with a strong olivine $(\mathrm{J}=16.2)$ and opx $(\mathrm{J}=13.3)$ LPO. We have taken the cpx LPO of sample 87VS28b from Barruol and Mainprice (1993) (Fig.10) to represent the upper mantle cpx component and included the effect of garnet on the velocity of the aggregate. To simulate the effect of composition we have used the Pyrolite and Piclogite models of Ita and Stixrude (1992). A strong LPO will give wide limits to the possible values as a function of orientation.
Using the single crystal pressure and temperature derivatives in Table 1 and geotherm given above, we have calculated the $\mathrm{Vp}$ anisotropy $\Phi=\left(\mathrm{V}_{\mathrm{PV}} / \mathrm{V}_{\mathrm{PH}}\right)^{2}$ as a function of depth assuming the lineation (X) and foliation (XY) plane was horizontal and pole to the foliation $(\mathrm{Z})$ was vertical (Fig. 14). From the arguments presented above, P-wave anisotropy should be more sensitive to the effects of composition in the upper mantle. We have plotted the anisotropic global models for comparison. The anisotropic petrological models correctly predict that $\mathrm{V}_{\mathrm{PH}}>\mathrm{V}_{\mathrm{PV}}$ in agreement with the global seismic models. Further, in the petrological models, the LPO strength is assumed to be constant with depth for each mineral, this results in a very gradual increase in $\left(\mathrm{V}_{\mathrm{PV}} / \mathrm{V}_{\mathrm{PH}}\right)^{2}$ with depth as is observed in the global models. Note the original PREM model has a slope in opposite sense which does not agree with more recent global models or the petrological models in Fig. 14. The gradual increase in $\left(\mathrm{V}_{\mathrm{PV}} / \mathrm{V}_{\mathrm{PH}}\right)^{2}$ with depth, which corresponds to a reduction in anisotropy, must be due to the variation of the elastic constants as a function of temperature and pressure along the geotherm as the LPO is constant for all phases and no compositional variation occurs before 250 $\mathrm{km}$ depth. The pyrolite model (Vp anisotropy of $8.7 \%$ and Vs anisotropy of $6.0 \%$ at $200 \mathrm{~km}$ ) is always more anisotropic than piclogite model $(\mathrm{AVp}=5.9 \%, \mathrm{AVs}=$ $3.9 \%$ ) because of the higher volume fraction of olivine (Fig. $5)$. Both models have same approximately orthorhombic symmetry (Fig. 15) with a strong variation in the $\mathrm{Vp}$ velocity in the foliation (XY) plane between a high velocity near the lineation $(\mathrm{X})$ and low velocity near Y. For this reason, we plotted $\Phi$ as $\left(\mathrm{V}_{\mathrm{PZ}} / \mathrm{V}_{\mathrm{PX}}\right)^{2}$ and $\left(\mathrm{V}_{\mathrm{PZ}} / \mathrm{V}_{\mathrm{PY}}\right)^{2}$ as the symmetry is not transverse isotropic as in the global seismic models with a unique velocity in the XY plane. The upper and lower bounds of a transverse isotropic petrological model are given by $\left(\mathrm{V}_{\mathrm{PZ}} / \mathrm{V}_{\mathrm{PX}}\right)^{2}$ and $\left(\mathrm{V}_{\mathrm{PZ}} / \mathrm{V}_{\mathrm{PY}}\right)^{2}$. The $\mathrm{P}-$ wave velocities are so similar in the $\mathrm{Y}$ and $\mathrm{Z}$ directions for the pyrolite model that it gives $\Phi=1.0$, the isotropic value. To estimate the transverse isotropic (TI) petrological models we have taken the arithmetic mean of the upper and lower bounds (Fig. 14). The TI petrological models are more anisotropic than the global models, which could be caused by many factors including heterogeneity which is not incorporated in this very simple anisotropic scheme. Heterogeneity on the seismic scale is equivalent to microstructure on the sample scale (see above the statistical continuum approach to microstructural description), it will cause the effective property (TI petrological estimate in this case) to vary between the bounds as a function of orientation. Theoretically it is possible that the TI petrological estimate could be closer to the lower bound and hence the global models if heterogeneity were incorporated. Finally, we modified the Pyrolite and Piclogite models of Ita and Stixrude (1992) to include the transformation of opx to cpx at $300 \mathrm{~km}$ as suggested by several experimental studies 


\section{\begin{tabular}{c} 
PETROLOGICAL MODEL : \\
SEISMIC ANISOTROPY AT $200 \mathrm{~km}$ \\
\hline
\end{tabular}}
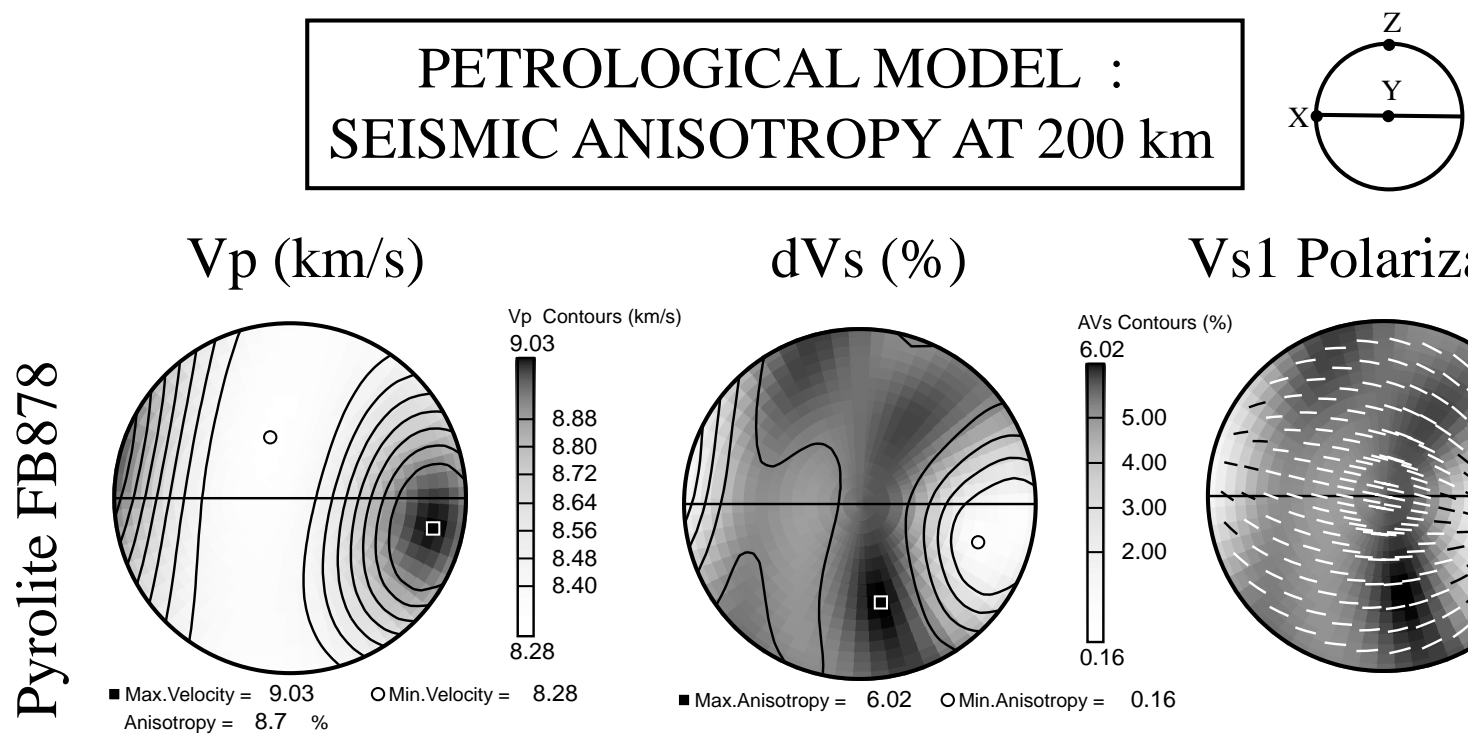

Vs1 Polarization

.
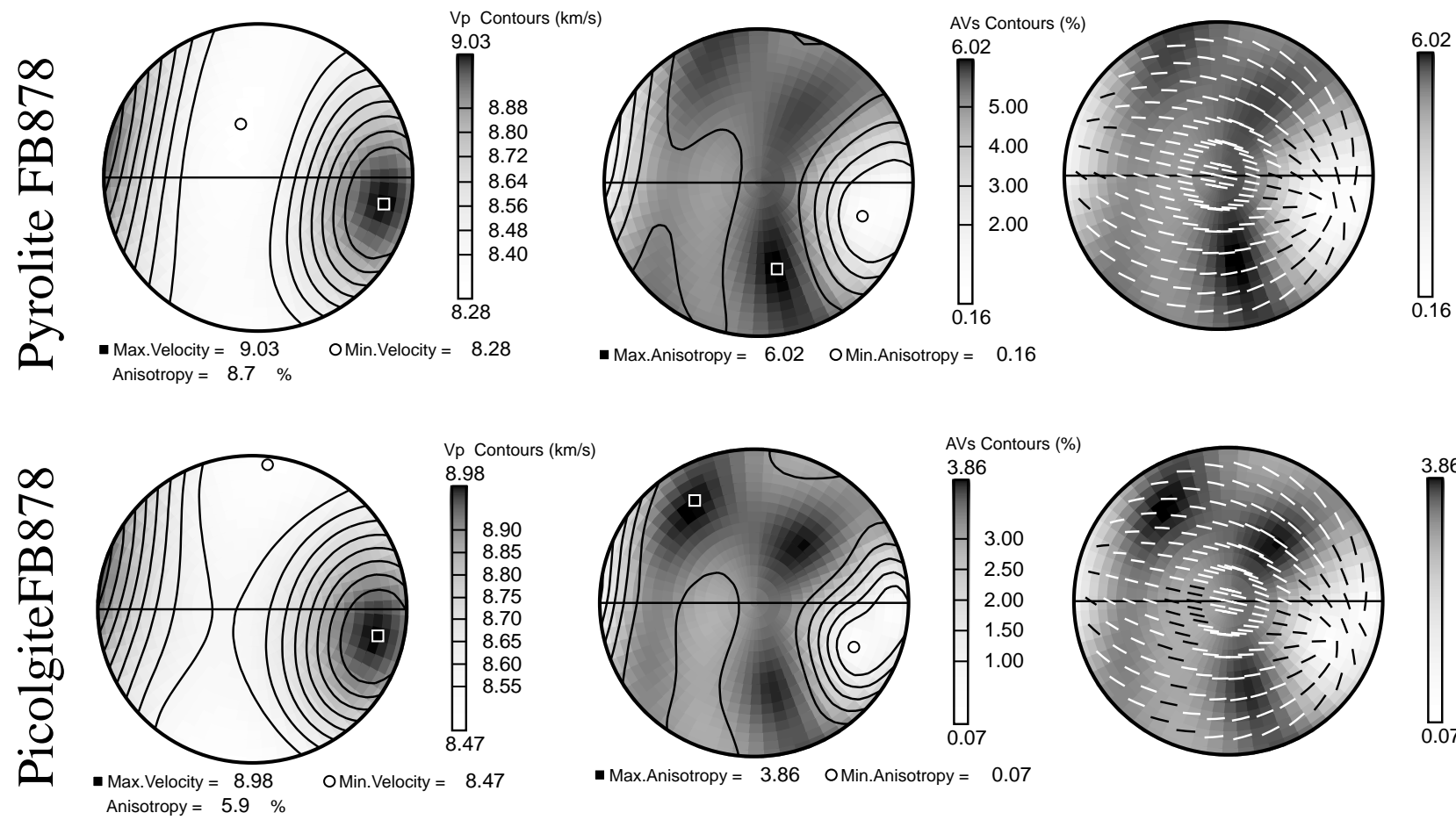

Figure 15. Seismic anisotropy of the pyrolite and piclogite petrological compositions at $200 \mathrm{~km}$ depth. Contours for $\mathrm{Vp}$ in $\mathrm{km} / \mathrm{s}, \mathrm{dVs}$ in $\%$ anisotropy and trace of the Vs1 polarization plane.

(Kanzaki, 1991; Pacalo and Gasparik, 1990; Woodland and Angel, 1997). Note that the transformation involves the opx (space group Pbca) to the so-called high pressure form of cpx (space group C2/c) (see Angel and Hugh-Jones, 1994). The elastic constants of the low pressure form of cpx (space group $\mathrm{P} 21 / \mathrm{c}$ ) have not been measured. The result is that both

models become slightly more anisotropic ( $\Phi$ decreases) below $300 \mathrm{~km}$. The anisotropy change is due to the velocity changes on the removal of opx. In $\mathrm{X}$ and $\mathrm{Z}$ directions, there is an overall reduction in $\mathrm{Vp}$ whereas in the $\mathrm{Y}$ direction it increases causing the $\mathrm{Z} / \mathrm{X}$ ratio to be nearly constant and the $\mathrm{Z} / \mathrm{Y}$ to decrease. These predictions are sensitive to the temperature and pressure derivatives of cpx which have not yet been experimentally measured. In the limit of the available data, the LPO strength to $200 \mathrm{~km}$ seems to be constant, the tendency of the global models towards isotropy at $350 \mathrm{~km}$ appears to be due to a real reduction in LPO strength between 200 and $350 \mathrm{~km}$ (e.g. Karato, 1992) rather than the opx to cpx transformation. Both the Pyrolite and Piclogite models are consistent with PV/PH symmetry of global models, although they predict a TI model which has a stronger anisotropy than the global models.

\section{CONCLUSIONS}

In this review, we have set out to describe the recent changes to our thinking about the Earth's mantle caused by the increase in knowledge of the mineral physics of single crystal properties and how they influence our ability to interpret anisotropic seismic data. The single crystal data can now be used to calculate polycrystal properties in a fairly standard way. Recently developments have allowed the incorporation of oriented melt phases in anisotropic medium which should allow more realistic modeling of structures such as mid ocean ridges. Our understanding of the upper mantle has increased considerably due to the fact we can now establish a statistical view of LPO, which is still far from complete, but at least for olivine and opx allows us to be very confident in our conclusions. Data is lacking for cpx, firstly the temperature and pressure derivatives of the elastic constants need to be experimentally measured, secondly very few LPO data are available for mantle samples of diopside. Such data are required for more rigorous testing of anisotropic petrological models. Despite these problems, a relatively simple picture of the symmetry of anisotropy for 
the upper mantle prevails because the dominant phases are olivine and opx. For example, both the Pyrolite and Piclogite models give similar anisotropy patterns which is due to the relatively mild interference of the $\mathrm{Vp}$ of the two minerals which results in a decrease in anisotropy and increased tendency for a transition from orthorhombic to transverse isotropic symmetry. The $\mathrm{Vp}$ maximum is always near the lineation $(\mathrm{X})$. The $\mathrm{dVs}$ anisotropy of the two minerals is constructive and results in a Vs1 polarization parallel to the foliation plane and a maximum $\mathrm{dVs}$ somewhere in $\mathrm{YZ}$ plane, most often near Y. All these features are extremely robust. The statistical approach to olivine LPO combined with recent experimental and numerical simulations of LPO show that the olivine LPO intensity ( $\mathrm{J}$ index) only records shear strain of less than 2.5 gamma. LPO simulations used for geodynamic modeling need to take into account this saturation in intensity, almost certainly due to recrystallization, although the effect on seismic anisotropy derived from simulations is likely to be very minor. Comparison between global seismic and anisotropic petrological models show that the LPO strength to $200 \mathrm{~km}$ seems to be constant with a decrease in LPO strength between 200 and $350 \mathrm{~km}$. There appears to be no seismic evidence for the opx to cpx transformation from the anisotropic component of global models.

In the transition zone and lower mantle the single crystal elastic constants and pressure derivatives are now determined either by experiment or theoretical calculation. However, very few temperature derivatives of the single crystal have been determined. Deformation experiments are now possible in transition zone conditions (e.g. Green et al., 1992; Sharp et al., 1994; Karato et al., 1998) which should allow a better documentation of the slip systems, deformation mechanisms and LPO development. Current global seismic models cannot be interpreted in terms of LPO anisotropy for this depth range, suggesting a possible layering at least at the bottom of the transition zone.

The lower mantle remains a vast region of exploration for mineral physics and seismology where many interpretations remain very speculative. The deformation mechanisms of silicate perovskite still remains an important objective. To date, much work have been achieved with oxide analogues. The anisotropy at the top of the lower mantle could be explained by plastic deformation of perovskite (Karato, 1998), but other factors such as the highly anisotropic $\mathrm{SiO}_{2}$ polymorphs could play a role in this

depth range. However, there is no experimental work on the deformation of these polymorphs and given their limited volume fraction, they may be oriented by shape control via viscous rather than plastic flow. The major feature of the lower mantle is however the $1600 \mathrm{~km}$ of virtually isotropic material from 1000 to $2600 \mathrm{~km}$ depth which is apparently seismically featureless. The isotropic lower mantle has been interpreted as zone of diffusive or superplastic creep which best fits our current mineral physics understanding of the problem (Karato et al., 1995).

Acknowledgments. We thank Andrea Tommasi for her figure on the strength of LPO as a function of finite strain and helpful comments on the manuscript. We thank Professor Jean-Paul Montagner for providing his global seismic models in computer readable form and discussions. We thank Françoise Boudier, Adolphe Nicolas and Paul Silver for their continued encouragement to make a more quantitative approach to upper mantle LPO development and seismic anisotropy. We apologize in advance for to all the authors of papers published on this subject before the Nicolas and Christensen 1987 AGU review paper which we have not cited due to lack of space and the many others we could have also cited. Finally we thank Professor S.-I. Karato for providing the occasion to write this review. Comments by S.-I. Karato and L. Stixrude helped improve the final version of the manuscript.

\section{REFERENCES}

Abramson, E.H., J.M. Brown, L.J. Slutsky and J. Zang, J., The elastic constants of San Carlos olivine to $17 \mathrm{Gpa}$, , J. Geophys. Res., 102, 12253-12263, 1997.

Adams, B.L., S.L. Wright, and K. Kunze, Orientation mapping: The emergence of a new microscopy, Metal. Trans. A24, 819-831, 1993.

Aleksandrov, K.S., T.V. Ryzhova and B.P. Belikov, The elastic properties of pyroxenes, Sov. Phys. Crystallogr., 8, 589-591, 1964.

Anderson, D.L., Deep structure of the continents, $J$. Geophys. Res., 84, 7555-7560, 1979.

Angel, R.J., and D.A. Hugh-Jones, Equations of state and thermodynamic properties of enstatite pyroxenes, $J$. Geophys. Res., 99, 19777-19783, 1994.

Barruol, G., and H. Kern, P and S waves velocities and shear wave splitting in the lower crustal/upper mantle transition (Ivrea Zone). Experimental and calculated data, Phys. Earth Planet. Inter., 95, 175-194, 1996.

Barruol, G., and D. Mainprice, 3D seismic velocities calculated from LPOs and reflectivity of a lower crustal section - Example of the Val Sesia (Ivrea Zone, Northern Italy), Geophys. J. Int., 115, 1169-1188, 1993.

Bass, J.D., and D.L. Anderson, Composition of the upper mantle: geophysical tests of two petrological models, Geophys. Res. Lett., 11, 229-232, 1984.

Ben Ismaïl, W., and D. Mainprice, An olivine fabric database: an overview of upper mantle fabrics and seismic anisotropy, Tectonophysics, 296, 145-157, 1998.

Ben Ismaïl, W., and D. Mainprice, An orthopyroxene fabric database: implications for seismic anisotropy, Tectonophysics, submitted, 1999.

Beran, M.J., Statistical continuum theories, John Wiley and Sons Interscience, New York, 1968.

Beran, M.J., T.A. Mason, B.L. Adams, and T. Olsen, Bounding elastic constants of an orthotropic polycrystal using measurements of the microstructure, J. Mech. Phys. Solids, 44, 1543-1563, 1996. 
Blackman, D.K., and J.-M. Kendall, Sensitivity of teleseismic body waves to mineral texture and melt in the mantle beneath a mid-ocean ridge, Phil. Trans. $R$. Soc. Lond. A, 355, 217-231, 1997.

Blackman, D.K., J.-M. Kendall, P. Dawson, H.-R. Wenk, D. Boyce, and J. Phipps Morgan, Teleseismic imaging of subaxial flow at mid-ocean ridges: travel-time effects of anisotropic mineral texture in the mantle, Geophys. $J$. Int., 127, 415-426, 1996.

Boyd, F.R., A pyroxene geotherm, Geochem. Cosmochim. Acta, 37, 2533-2546, 1973.

Brown, J.M., and T.J. Shankland, Thermodynamic parameters in the Earth as determined from seismic profiles, Geophys. J. R. Astr. Soc., 66, 579-596, 1981.

Bruner, W.M., Comments on "Seismic velocities in dry and saturated cracked solids", J. Geophys. Res., 81, 25732576, 1976.

Bunge, H.J., Texture analysis in materials sciences, 593 pp., Buttleworth, London, 1982.

Chai, M., J.M. Brown, and L.J. Slutsky, The elastic constants of a pyrope-grossular-almandine garnet to 20 GPa, Geophys. Res. Lett., 24, 523-526, $1997 \mathrm{a}$.

Chai, M., J.M. Brown, and L.J. Slutsky, The elastic constants of an aluminous orthopyroxene to $12.5 \mathrm{GPa}$, J. Geophys. Res., 102, 14779-14785, 1997b.

Chastel, Y.B., P.R. Dawson, H.-R. Wenk, and K. Bennet, Anisotropic convection with implications for the upper mantle, J. Geophys. Res., 98, 17757-17771, 1993.

Collins, M.D. and J.M. Brown, Elasticity of an upper mantle clinopyroxene, Phys. Chem. Miner., 26, 7-13.

Crampin, S., Effective anisotropic elastic constants for wave propagation through cracked solids, Geophys. J. $R$. Astron. Soc., 76, 135-145, 1984.

Crosson, R.S., and J.W. Lin, Voigt and Reuss prediction of anisotropic elasticity of dunite, J. Geophys. Res., 76, 570-578, 1971.

Da Silva, C.R.S., Karki, B.B., Stixrude, L., and Wentzcovitch, R.M. Ab initio study of the elastic behavior of $\mathrm{MgSiO} 3$ ilmenite at high pressure. Geophys. Res. Lett., 26, 943-946, 1999.

Duffy, T.S. and M.T. Vaughan, Elasticity and its relationship to crystal structure, , J. Geophys. Res., 93, 383-391, 1988.

Duffy, T.S., and D.L. Anderson, Seismic velocities in mantle minerals and the mineralogy of the upper mantle, J. Geophys. Res., 94, 1895-1912, 1989.

Dupas-Bruzek, C., T. Sharp, D.C. Rubie, and W.B. Durham, Mechanisms of transformation and deformation in $\mathrm{Mg} 1.8 \mathrm{Fe} 0.2 \mathrm{SiO} 4$ olivine and wadsleyite under nonhydrostatic stress, Phys. Earth Planet. Inter., 108, 33-48, 1998.

Dziewonski, A.M., and D.L. Anderson, Preliminary reference Earth model, Phys. Earth Planet. Inter., 25, 297-356, 1981.

Eshelby, J.D., The determination of the elastic field of a ellipsoidal inclusion, and related problems, Proc. $R$. Soc. London, 241, 376-396, 1957.

Estey, L.H., and B.J. Douglas, Upper mantle anisotropy: A preliminary model, J. Geophys. Res., 91, 11393-11406, 1986.
Etchecopar, A., and G. Vasseur, A 3-D kinematic model of fabric development in polycrystalline aggregates: comparison with experimental and natural examples, $J$. Struct. Geol., 9, 705-717, 1987.

Fei, Y., Thermal expansion, in Minerals physics and crystallography: a handbook of physical constants, edited by T.J. Ahrens, pp. 29-44, American Geophysical Union, Washington D.C., 1995.

Flanagan, M.P., and P.M. Shearer, Global mapping of topography on transition zone velocity discontinuities by stacking SS precursors, J. Geophys. Res., 103, 26732692, 1998.

Frisillo, A.L., and G.R. Barsch, Measurement of singlecrystal elastic constants of bronzite as a function of pressure and temperature, J. Geophys. Res., 77, 63606384, 1972.

Gaherty, J.B., and T.H. Jordan, Lehmann discontinuity as the base of an anisotropic layer beneath continents, Science, 268, 1468-1471, 1995.

Green, H.W., Young, T.E., Walker, D., and Scholtz, C.H., The effect of non hydrostatic stress on the $\alpha-\beta$ olivine phase transformations, in High-Pressure Research: Application to Earth and Planetary Sciences, edited by Y. Syono and M. N. Manghnani, pp. 229-235, American Geophysical Union, Washington D.C., 1992.

Gillet, P., Mineral physics, mantle mineralogy and mantle dynamics, C.R. Académie des Sciences, 320 série IIa, 341-356, 1995.

Godard, G., and H.L.M. van Roermund, Deformationinduced clinopyroxene fabrics from eclogites, J. Struct. Geol., 17, 1425-1443, 1995.

Haggerty, S.E., and V. Sautter, Ultra deep (>300 km) ultramafic, upper mantle xenoliths, Science, 248, 993996, 1990.

Harte, B., and J.W. Harris, Lower mantle inclusions from diamonds, Terra Nova, 5, 101, 1993.

Heinemann, S., T.G. Sharp, F. Seifert and D.C. Rubie, The cubic-tetragonal phase transition in the system majorite $\left(\mathrm{Mg}_{4} \mathrm{Si}_{4} \mathrm{O}_{12}\right)$ - pyrope $\left(\mathrm{Mg}_{3} \mathrm{Al}_{2} \mathrm{Si}_{3} \mathrm{O}_{12}\right)$, and garnet symmetry in the Earth's transition zone, Phys. Chem. Miner., 24, 206-221, 1997.

Helffrich, G.R., and B.J. Wood, $410 \mathrm{~km}$ discontinuity sharpness and the form of the olivine $\alpha-\beta$ phase diagram: resolution of apparent seismic contradictions, Geophys. J. Int., 126, 7-12, 1996.

Henyey, F.S., and N. Pomphrey, Self-consistent elastic moduli of a cracked solid, Geophys. Res. Lett., 9, 903906, 1982.

Hess, H.H., Seismic anisotropy of the uppermost mantle under oceans, Nature, 203, 629-631, 1964.

Hill, R., The elastic behaviour of a crystalline aggregate, Proc. Phys. Soc. London Sec., 65, 349-354, 1952.

Hill, R., A self consistent mechanics of composite materials, J. Mech. Phys. Solids, 13, 213-222, 1965.

Irifune, T., Absence of an aluminous phase in the upper part of the Earth's lower mantle, Nature, 370, 131-133, 1994.

Ita, J., and L. Stixrude, Petrology, elasticity and composition of the mantle transition zone, J. Geophys. Res., 97, 6849-6866, 1992.

Issak, D.G., High-temperature elasticity of iron-bearing olivine, J. Geophys. Res., 97, 1871-1885, 1992. 
Issak, D.G., O.L., Anderson, and T. Goto, Measured elastic moduli of single crystal $\mathrm{MgO}$ up to $1800 \mathrm{~K}$, Phys. Chem. Miner., 16, 704-713, 1989.

Ito, E., and T. Katsura, A temperature profile of the mantle transition zone, Geophys. Res. Lett., 16, 425-428, 1989.

Jackson, I. and H. Niesler, The elasticity of periclase to 3 Gpa and some geophysical implications, in High pressure research in geophysics, edited by S. Akimoto and M.H. Manghnani, pp 93-133, Center for Academic Publications, Tokyo, 1982.

Kanzaki, M., Ortho/Clinoenstatite transition, Phys. Chem. Miner., 17, 726-730, 1991.

Karato, S.-I., On the Lehmann discontinuity, Geophys. Res. Lett., 19, 2255-2258, 1992.

Karato, S.-I., Seismic anisotropy in the deep mantle, boundary layers and the geometry of mantle convection, Pure Appl. Geophys., 151, 565-587, 1998.

Karato, S.-I., S. Zhang, and H.-R. Wenk, Superplastic in Earth's lower mantle: Evidence from seismic anisotropy and rock physics, Science, 270, 458-461, 1995.

Karato, S.-I., C. Dupas-Bruzek, and D.C. Rubie, Plastic deformation of silicate spinel under the transition zone conditions of the Earth's mantle, Nature, 395, 266-269, 1998.

Karki, B.B., L. Stixrude, S.J. Clark, M.C. Warren, G.J. Ackland, and J. Crain, Elastic properties of orthorhombic $\mathrm{MgSiO}_{3}$ perovskite at lower mantle pressures, $\mathrm{Am}$. Miner., 82, 635-638, 1997a.

Karki, B.B., L. Stixrude, and J. Crain, Ab initio elasticity of three high-pressure polymorphs of silica, Geophys. Res. Lett., 24, 3269-3272, 1997b.

Karki, B.B., L. Stixrude, S.J. Clark, M.C. Warren, G.J. Ackland, and J. Crain, Structure and elasticity of $\mathrm{MgO}$ at high pressure, Am. Miner., 82, 51-60, 1997c.

Kawakatsu, H., and F. Niu, Seismic evidence for a $920 \mathrm{~km}$ discontinuity in the mantle, Nature, 371, 301-305, 1994.

Kendall, J.M., and P.G. Silver, Constraints from seismic anisotropy on the nature of the lowermost mantle, Nature, 381, 409-412, 1996.

Kendall, J.M., and P.G. Silver, Investigating causes of D" anisotropy, in The Core-Mantle Boundary Region, edited by M. Gurnis, M. Wysession, E. Knittle, and B. Buffet, pp. 409-412, American Geophysical Union, Washington, D.C., 1998.

Kesson, S.E., and J.D. Fitz Gerald, Partitioning of $\mathrm{MgO}$, $\mathrm{FeO}, \mathrm{NiO}, \mathrm{MnO}$ and $\mathrm{Cr}_{2} \mathrm{O}_{3}$ between magnesian silicate perovskite and magnesiowüstite: implications for the inclusions in diamond and the composition of the lower mantle, Earth Planet. Sci. Lett., 111, 229-240, 1991.

Kiefer, B., L. Stixrude, and R.M. Wentzcovitch, Calculated elastic constants and anisotropy of $\mathrm{Mg}_{2} \mathrm{SiO}_{4}$ spinel at high pressure, Geophys. Res. Lett., 24, 2841-2844, 1997.

Kind, R., G.L. Kosarev, L.I. Makeyeva, and L.P. Vinnik, Observation of laterally inhomogeneous anisotropy in the continental lithosphere, Nature, 318, 358-361, 1985.

Kingma, K.J., R.E. Cohen, R.J. Hemley and H.K. Mao, transformation of stishovite to a denser phase at lower mantle pressure, Nature, 374, 243-245, 1995.
Knittle, E., Static compression measurements of equation of state, in Minerals physics and crystallography: a handbook of physical constants, edited by T.J. Ahrens, pp. 98-142, American Geophysical Union, Washington D.C., 1995.

Kröner, E., Bounds for effective elastic moduli of disordered materials, J. Mech. Phys. Solids, 25, 137-155, 1977.

Kröner, E., Self-consistent scheme and graded disorder in polycrystal elasticity, J. Physics, 8, 2261-2267, 1978.

Kumazawa, M., The elastic constants of single crystal orthopyroxene., J. Geophys. Res., 74, 5973-5980, 1969.

Kumazawa, M. and O.L. Anderson, Elastic moduli, pressure derivatives and temperature derivatives of single crystal olivine and single crystal forsterite, J. Geophys. Res., 74, 5961-5980, 1969.

Lehmann, L., Velocities of longitudinal waves in the upper part of the Earth's mantle, Ann. Geophys., 15, 93-118, 1959.

Lehmann, I., $\mathrm{S}$ and the structure of the upper mantle, Geophys. J. R. Astron. Soc., 4, 124-138, 1961.

Leven, J., I. Jackson, and A.E. Ringwood, Upper mantle seismic anisotropy and lithosphere decoupling, Nature, 289, 234-239, 1981.

Levien, L., D.J. Weidner, and C.T. Prewitt, Elasticity of diopside, Phys. Chem. Miner., 4, 105-113, 1979.

Mainprice, D., Modeling anisotropic seismic properties of partially molten rocks found at mid-ocean ridges, Tectonophysics, 279, 161-179, 1997.

Mainprice, D., and M. Humbert, Methods of calculating petrophysical properties from lattice preferred orientation data, Surv. Geophys., 15, 575-592, 1994.

Mainprice, D., M. Humbert, and F. Wagner, Phase transformations and inherited lattice preferred orientation : Implications for seismic properties, Tectonophysics, 180, 213-228, 1990.

Mainprice, D., and A. Nicolas, Development of shape and lattice preferred orientations: application to the seismic anisotropy of the lower crust, J. Struct. Geol., 11, 175189, 1989.

Mason, T.A., and B.L. Adams, Use of microstructural statistics in predicting polycrystalline material properties, Metal. Trans. 30A, 969-979, 1999.

Matsui, M., and W.R. Busing, Calculation of the elastic constants and high-pressure properties of diopside, $\mathrm{CaMgSi}_{2} \mathrm{O}_{6}$, Am. Miner., 69, 1090-1095, 1984.

Matthies, S., and M. Humbert, The realization of the concept of a geometric mean for calculating physical constants of polycrystalline materials, Phys. Stat. Sol., 177, K47-K50, 1993.

Meade, C.P., P.G. Silver, and S. Kaneshima, Laboratory and seismological observations of lower mantle isotropy, Geophys. Res. Lett., 22, 1293-1296, 1995.

Mercier, J.-C., Single-pyroxene thermobarometry, Tectonophysics, 70, 1-37, 1980.

Mercier, J.C., Olivine and pyroxenes, in Preferred orientation in deformed metals and rocks: $A n$ introduction to modern texture analysis, edited by H.R. Wenk, pp. 407-430, Academic Press, Orlando, 1985.

Mercier, J.-C., and Carter, N.L. Pyroxene geotherms, J. Geophys. Res., 80, 3349-3362, 1975. 
Montagner, J.-P., What can seismology tell us about Mantle convection ?, Rev. Geophys., 32, 115-137, 1994.

Montagner, J.-P., Where can seismic anisotropy be detected in the Earth's mantle ? In boundary layers..., Pure Appl. Geophys., 151, 223-256, 1998.

Montagner, J.-P., and D.L. Anderson, Constraints on elastic combinations inferred from petrological models, Phys. Earth Planet. Int., 54, 82-105, 1989.

Montagner, J.-P., and B.L.N. Kennett, How to reconcile body-wave and normal-mode reference earth models, Geophys. J. Int., 125, 229-248, 1996.

Montagner, J.-P., and T. Tanimoto, Global anisotropy in the upper mantle inferred from the regionalization of phase velocities, J. Geophys. Res., 95, 4797-4819, 1990.

Moore, R.O., and J.J. Gurney, Pyroxene solid solution in garnets included in diamond, Nature, 335, 784-789, 1985.

Morawiec, A., Calculation of polycrystal elastic constants from single crystal data, Phys. Stat. Sol., 154, 535-541, 1989.

Morelli, A., A.M. Dziewonski, and J.H. Woodhouse, Anisotropy of the inner core inferred from PKiKP travel times, Geophys. Res. Lett., 13, 1545-1548, 1986.

Nataf, H.C., I. Nakanishi, and D.L. Anderson, Anisotropy and shear velocity heterogeneities in the upper mantle, Geophys. Res. Lett., 11, 109-112, 1984.

Nataf, H.C., I. Nakanishi, and D.L. Anderson, Measurements of mantle wave velocities and inversion for lateral inhomogeneities and anisotropy, J. Geophys. Res., 91, 7261-7307, 1986.

Nicolas, A., F. Boudier, and A.M. Boullier, Mechanism of flow in naturally and experimentally deformed peridotites, American Journal of Science, 273, 853-876, 1973.

Nicolas, A., and N.I. Christensen, Formation of anisotropy in upper mantle peridotites - A review, in Composition, Structure and Dynamics of the LithosphereAsthenosphere System, edited by K. Fuchs, and C. Froidevaux, pp. 111-123, AGU, Washington, D.C., 1987.

Ohno, I., Free vibration of rectangular parallelepiped crystal and its application to determination of elastic constants of orthorhombic crystals, J. Phys. Earth, 24, 355-379, 1976.

Pacalo, R.E.G., and T. Gasparik, Reversals of the orthoenstatite-clinoenstatite transition at high pressures and high temperatures, J. Geophys. Res., 95, 15853$15858,1990$.

Pacalo, R.E.G., and D.J. Weidner, Elasticity of majorite, $\mathrm{MgSiO}_{3}$ tetragonal garnet, Phys. Earth Planet. Inter., 99, 145-154, 1997.

Pacalo, R.E.G., D.J. Weidner, and T. Gasparik, Elastic properties of Sodium-rich Majorite garnet, Geophys. Res. Lett., 19, 1895-1898, 1992.

Polet, J., and D.L. Anderson, Depth extent of cratons as inferred from tomographic studies, Geology, 23, 205208, 1995.

Raitt, R.W., G.G. Schor, T.J.G. Francis, and G.B. Morris, Anisotropy of the Pacific upper mantle, J. Geophys. Res., 74, 3095-3109, 1969.
Reuss, A., Berechnung der von Mischkristallen auf Grund der Plastizitätsbedingung für Einkristalle, Z. Angew. Math. Mech., 9, 49-58, 1929.

Ringwood, A.E., Phase transitions and their bearing on the constitution and dynamics of the mantle, Geochimica et Cosmochimica Acta, 55, 2083-2110, 1991.

Sautter, V., S.E. Haggerty, and S. Field, Ultra deep (>300 $\mathrm{km})$ xenoliths : new petrological evidence from the transition zone, Science, 252, 827-830, 1991.

Savage, M.K., Seismic anisotropy and Mantle deformation: What have we learned from shear wave splitting? Ann. Rev. Earth Planet. Sci., in press, 1999.

Sawamoto, H., D.J. Weidner, S. Sasaki, and M. Kumazawa, Single-crystal elastic properties of the modified-spinel (beta) phase of magnesium orthosilicate, Science, 224, 749-751, 1984.

Scott-Smith, B.H., R.V. Danchin, J.W. Harris, and K.J. Stracke, Kimberlites near Orrorroo, South Australia, in Kimberlites I: Kimberlites and related rocks, edited by J. Kornprobst, pp. 121-142, Elsevier, Amsterdam, 1984.

Sharp, T.G., Bussod, G.Y.A., and T. Katsura, Microstructures in b-Mg1.8Fe0.2SiO4 experimentally deformed at transition-zone conditions, Phys. Earth Planet. Inter., 86, 69-83, 1994.

Shearer, P.M., Transition zone velocity gradients and the $520 \mathrm{~km}$ discontinuity, J. Geophys. Res., 101, 30533066, 1996.

Silver, P.G., Seismic anisotropy beneath the continents: Probing the depths of geology, Ann. Rev. Earth Planet. Sci., 24, 385-432, 1996.

Silver, P.G., and W. Chan, Implications for continental structure and evolution from seismic anisotropy., Nature, 335, 34-39, 1988.

Silver, P.G., and W.W. Chan, Shear wave splitting and subcontinental mantle deformation, J. Geophys. Res., 96, 16429-16454, 1991.

Sinogeikin, S.V., T. Katsura, and J.D. Bass, Sound velocities and elastic properties of Fe-bearing wadsleyite and ringwoodite, J. Geophys. Res., 103, 20819-20825, 1998.

Tommasi, A., D. Mainprice, G. Canova, and Y. Chastel, Viscoplastic self-consistent and equilibrium-based modeling of olivine lattice preferred orientations. 1.Implications for the upper mantle seismic anisotropy, J. Geophys. Res., submitted, 1999.

Vinnik, L., S. Chevrot, and J.P. Montagner, Evidence for a stagnant plume in the transition zone?, Geophys. Res. Lett., 24, 1007-1010, 1997.

Vinnik, L.P., R. Kind, G.L. Kosarev, and L.I. Makeyeva, Azimuthal anisotropy in the lithosphere from observations of long-period S-waves, Geophys. J. Int., 99, 549-559, 1989.

Vinnik, L.P., and J.P. Montagner, Shear wave splitting in the mantle PS phases, Geophys. Res. Lett., 23, 24492452, 1996.

Voigt, W., Lerrbuch der Kristallphysik, Teubner-Verlag, Leipzig, 1928.

Watt, J.P., Elastic properties of polycrystalline materials: Comparison of theory and experiment, Phys. Chem. Miner., 15, 579-587, 1988. 
Webb, S.L., The elasticity of the upper mantle orthosilicates olivine and garnet to $3 \mathrm{Gpa}$, Phys. Chem. Miner., 16, 684-692, 1989.

Webb, S.L., and I. Jackson, The pressure dependence of the elastic moduli of single-crystal orthopyroxene $\left(\mathrm{Mg}_{0.8} \mathrm{Fe}_{0.2}\right) \mathrm{SiO}_{3}$, European Journal of Mineralogy, 5, 1111-1119, 1993.

Weidner, D.J., A mineral physics test of a pyrolite mantle, Geophys. Res. Lett., 12, 417-420, 1985.

Weidner, D.J., Mantle models based on measured physical properties of minerals, in Chemistry and physics of terrestrial planets, edited by S.K. Saxena, pp. 251-274, Springer, Berlin, 1986.

Weidner, D.J., J.D. Bass, A.E. Ringwood, and W. Sinclair, The single-crystal elastic moduli of stishovite, J. Geophys. Res., 87, 4740-4746, 1982.

Weidner, D.J., and E. Ito, Elasticity of $\mathrm{MgSiO}_{3}$ in the ilmenite phase, Phys. Earth Planet. Inter., 40, 65-70, 1985.

Weidner, D.J., H. Sawamoto, S. Sasaki, and M. Kumazawa, Single-crystal elastic properties of the spinel phase of $\mathrm{Mg}_{2} \mathrm{SiO}_{4}$, J. Geophys. Res., 89, 7852-7860, 1984.

Weidner, D.J., H. Wang, and J. Ito, Elasticity of orthoenstatite, Phys. Earth Planet. Inter., 17, 7-13, 1978.

Wenk, H.-R., K. Bennet, G.R. Canova, and A. Molinari, Modeling plastic deformation of peridotite with the selfconsistent theory, J. Geophys. Res., 96, 8337-8349, 1991.

Wenk, H.-R., G.R. Canova, Y. Brechet, and L. Flandin, A deformation-based model for recrystallization of anisotropic materials, Acta Metallurgica Materialia, 45, 3283-3296, 1997.

Williams, Q., and E.J. Garnero, Seismic evidence for partial melt at the base of the Earth's mantle, Science, 273, 1528-1530, 1996.

Woodland, A.B., and R.J. Angel, Reversal of the orthoferrosilite high-P clinoferrosilite transition, a phase diagram for $\mathrm{FeSiO}_{3}$ and implications for the mineralogy of the Earth's upper mantle, European Journal of Mineralogy, 9, 245-254, 1997.

Yeganeh-Haeri, A., Synthesis and re-investigation of the elastic properties of single-crystal magnesium silicate perovskite, Phys. Earth Planet. Inter., 87, 111-121, 1994.

Yeganeh-Haeri, A., D.J. Weidner, and E. Ito, Elasticity of $\mathrm{MgSiO}_{3}$ in the perovskite structure, Science, 243, 787789, 1989.

Zha, C.-s., T.S. Duffy, H.-k. Mao, R.T. Downs, R.J. Hemley, and D.J. Weidner, Single-crystal elasticity of $\beta$ $\mathrm{Mg}_{2} \mathrm{SiO}_{4}$ to the pressure of the $410 \mathrm{~km}$ seismic discontinuity in the Earth's mantle, Phys. Earth Planet. Inter., 147, 9-15, 1997.

Zhang, S., and S. Karato, Lattice preferred orientation of olivine aggregates in simple shear, Nature, 375, 774$777,1995$.

David Mainprice, Guilhem Barruol and Walid Ben Ismaïl, Laboratoire de Tectonophysique, CNRS, Université de Montpellier II, 34095 Montpellier cedex 05, France. (email: mainprice@dstu.univ-montp2.fr, barruol@dstu.univ-montp2.fr, walid@dstu.univ-montp2.fr) 